\title{
Organometallic Complexes as Hole Transporting Materials in Organic Light Emitting Diodes
}

\author{
Xiaofan Ren, Bert D. Alleyne, Peter I. Djurovich, Chihaya Adachi ${ }^{\ddagger}$, Irina Tsyba, Robert Bau, \\ Mark Thompson* \\ Department of Chemistry, University of Southern California, Los Angeles, CA 90089
}

\section{Supporting Information}

Figure S 1. Voltage vs time (top) and luminescence vs time (bottom).Current - voltage and brightness - voltage curves (top) and external quantum efficiency-voltage curves (bottom) for $f a c-\mathrm{Co}(p p z)_{3}$ and NPD OLEDs. The device structure was ITO/ fac-Co $(p p z)_{3}$ $(400 \AA) / \mathrm{Gapma}(100 \AA) / \mathrm{Alq}_{3}(500 \AA) / \mathrm{Mg}: \mathrm{Ag}$.

Figure S 2. Current Current - voltage and brightness - voltage curves (top) and external quantum efficiency - voltage curves (bottom) for $f a c-C o(p p y)_{3}$ and NPD OLEDs. The device structure was ITO/ fac-Co(ppy) $3 /(400 \AA) / \mathrm{Gapma}(100 \AA) / \mathrm{Alq}_{3}(500 \AA) / \mathrm{Mg}: \mathrm{Ag}$.

Figure S 3. HOMO and LUMO isosurface plots of $f a c-\mathrm{Co}(p p z)_{3}, f a c-\mathrm{Co}(p p y)_{3}$ and Gapma.

Figure S 4. Ortep plots for $f a c-\operatorname{Co}(p p z)_{3}$, Gapma and $\operatorname{Co}\left(p p z_{2}\right) \mathrm{Cl}_{2}$.

Table S 1. Atomic coordinates ( $\left.\mathrm{x} 10^{4}\right)$ and equivalent isotropic displacement parameters $\left(\AA^{2} \mathrm{x}\right.$ $\left.10^{3}\right)$ for $f a c-\mathrm{Co}(p p z)_{3} \cdot 0.5 \mathrm{CH}_{2} \mathrm{Cl}_{2}$. U(eq) is defined as one third of the trace of the orthogonalized Uij tensor.

Table S 2. Bond lengths $[\AA]$ and angles $\left[^{\circ}\right]$ for $f a c-\mathrm{Co}(p p z)_{3} \cdot 0.5 \mathrm{CH}_{2} \mathrm{Cl}_{2}$.

Table S 3. Anisotropic displacement parameters $\left(\AA^{2} \times 10^{3}\right)$ for $f a c-\mathrm{Co}(p p z)_{3} \cdot 0.5 \mathrm{CH}_{2} \mathrm{Cl}_{2}$. The anisotropic displacement factor exponent takes the form: $-2 \pi^{2}\left[\mathrm{~h}^{2} \mathrm{a}^{* 2} \mathrm{U}^{11}+\ldots+2 \mathrm{~h} \mathrm{k} \mathrm{a}^{*} \mathrm{~b}^{*}\right.$ $\left.\mathrm{U}^{12}\right]$.

Table S 4. Hydrogen coordinates ( $\left.\times 10^{4}\right)$ and isotropic displacement parameters $\left(\AA^{2} \times 10^{3}\right)$ 
for $f a c-\mathrm{Co}(p p z)_{3} \cdot 0.5 \mathrm{CH}_{2} \mathrm{Cl}_{2}$.

Table S 5. Atomic coordinates ( x 104) and equivalent isotropic displacement parameters $\left(\AA^{2} \mathrm{x}\right.$ $10^{3}$ )for Gapma. U(eq) is defined as one third of the trace of the orthogonalized Uij tensor.

Table S 6. Bond lengths $[\AA]$ and angles $\left[^{\circ}\right]$ for Gapma.

Table S 7. Anisotropic displacement parameters $\left(\AA^{2} \times 10^{3}\right)$ for Gapma. The anisotropic displacement factor exponent takes the form: $-2 \pi^{2}\left[h^{2} a^{* 2} U^{11}+\ldots+2 h k a^{*} b^{*} U^{12}\right]$.

Table S 8. Hydrogen coordinates ( x 104) and isotropic displacement parameters $\left(\AA^{2} \times 10^{3}\right)$ for Gapma.

Table S 9. Atomic coordinates ( x 104) and equivalent isotropic displacement parameters $\left(\AA^{2} \mathrm{X}\right.$ $10^{3}$ ) for $\mathrm{Co}\left(p p z_{2}\right) \mathrm{Cl}_{2}$. U(eq) is defined as one third of the trace of the orthogonalized Uij tensor.

Table S 10. Bond lengths $[\AA]$ and angles $\left[^{\circ}\right]$ for $\operatorname{Co}\left(p p z_{2}\right) \mathrm{Cl}_{2}$.

Table S 11. Anisotropic displacement parameters $\left(\AA^{2} \times 10^{3}\right)$ for $\operatorname{Co}\left(p p z_{2}\right) \mathrm{Cl}_{2}$. The anisotropic displacement factor exponent takes the form: $-2 \pi^{2}\left[h^{2} a^{* 2} U^{11}+\ldots+2 h k a^{*} b^{*} U^{12}\right]$.

Table S 12. Hydrogen coordinates ( $\left.\mathrm{x} 10^{4}\right)$ and isotropic displacement parameters $\left(\AA^{2} \mathrm{x} 10^{3}\right)$ for $\mathrm{Co}\left(p p z_{2}\right) \mathrm{Cl}_{2}$. 
Figure S 1. Voltage vs time (top) and luminescence vs time (bottom).Current - voltage and brightness - voltage curves (top) and external quantum efficiency-voltage curves (bottom) for $f a c-C o(p p z) 3$ and NPD OLEDs. The device structure was ITO/ fac-Co(ppz)3
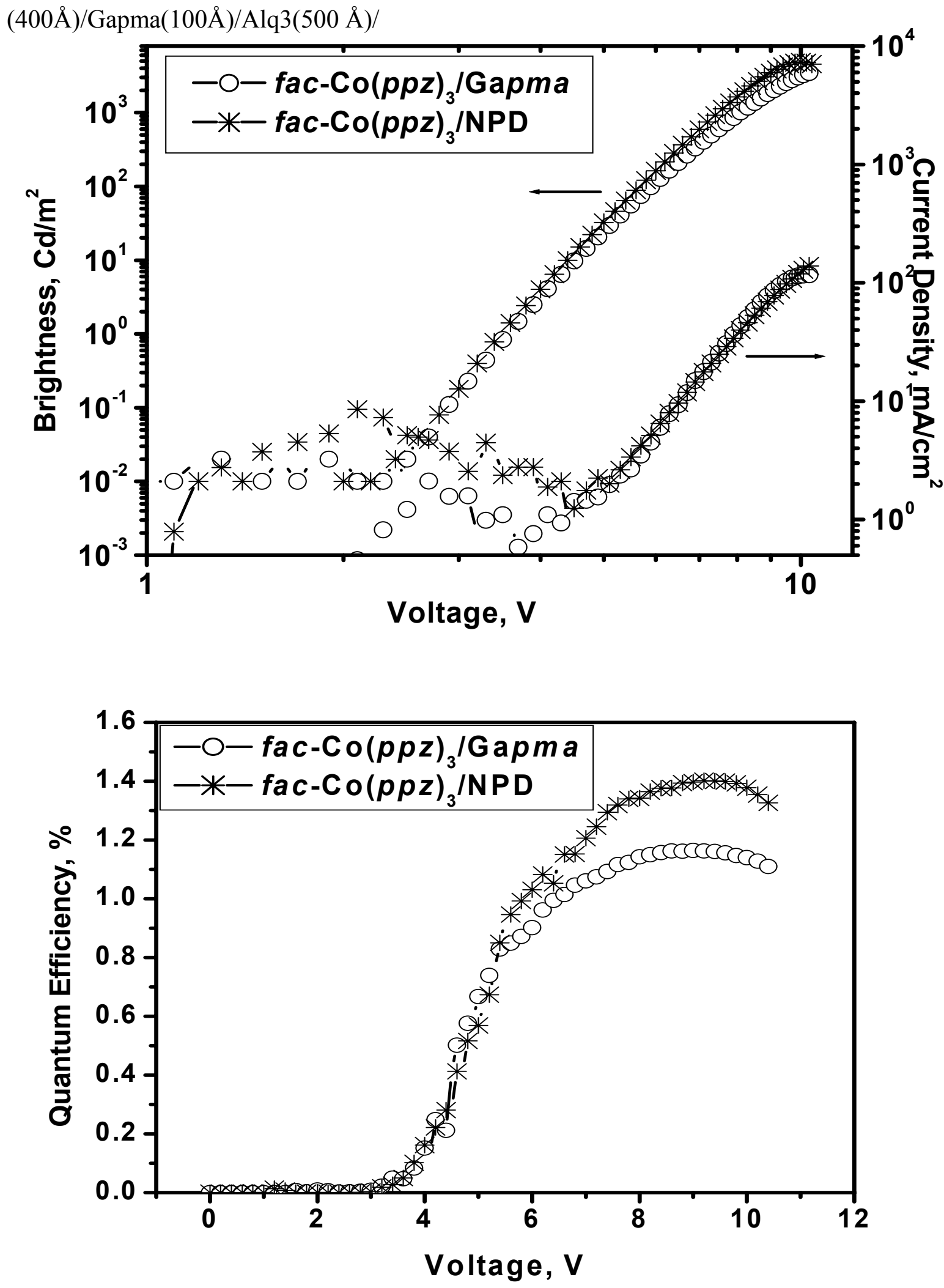
Figure S 2. Current Current - voltage and brightness - voltage curves (top) and external quantum efficiency - voltage curves (bottom) for $f a c-\operatorname{Co}(p p y)_{3}$ and NPD OLEDs. The device structure was ITO/fac-Co $(p p y)_{3} /(400 \AA) / \mathrm{Gapma}(100 \AA) / \mathrm{Alq}_{3}(500 \AA) / \mathrm{Mg}: \mathrm{Ag}$.
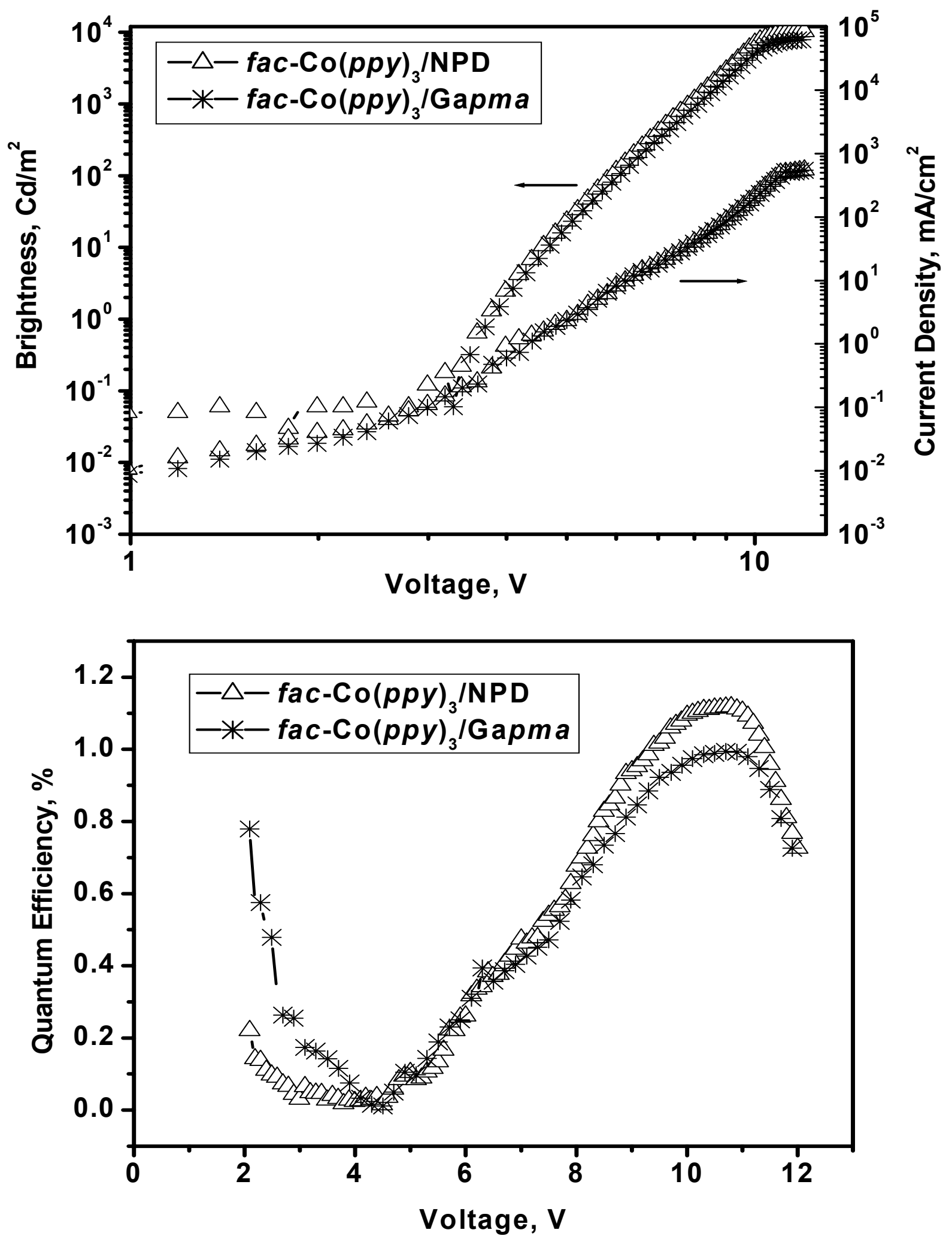
Figure S 3. HOMO and LUMO isosurface plots of $f a c-\mathrm{Co}(p p z)_{3}, f a c-\mathrm{Co}(p p y)_{3}$ and Gapma.

$$
f a c-C o(p p z)_{3}
$$

HOMO (-5.45 eV)

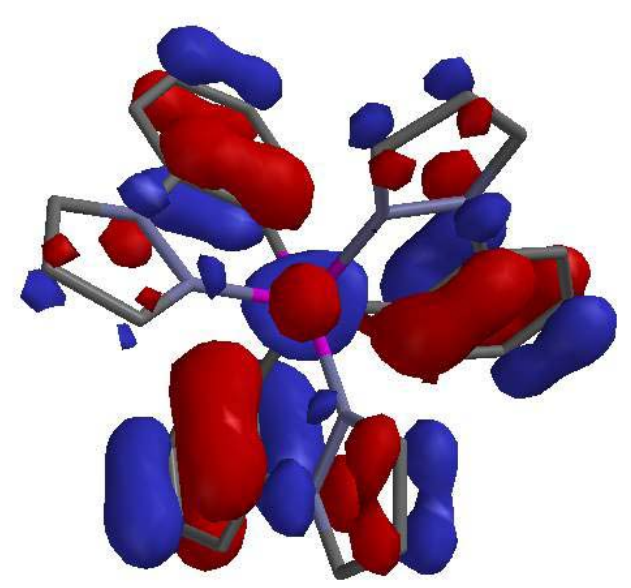

LUMO (-0.52 eV)

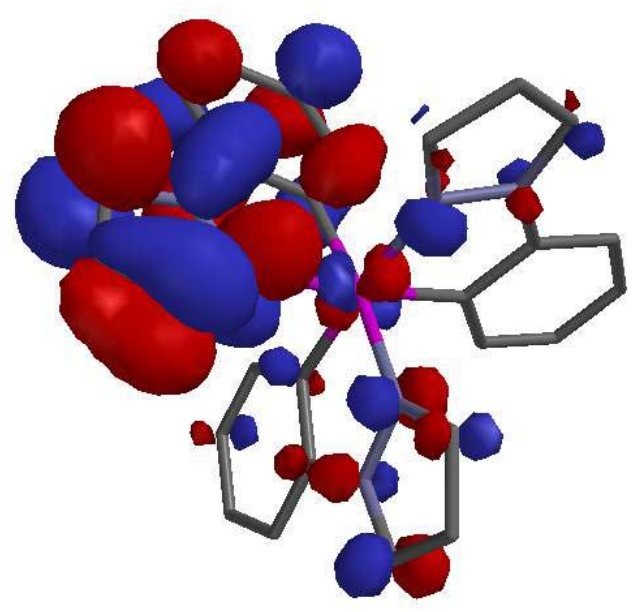

$f a c-\operatorname{Co}(p p y)_{3}$

LUMO (-1.17 eV)

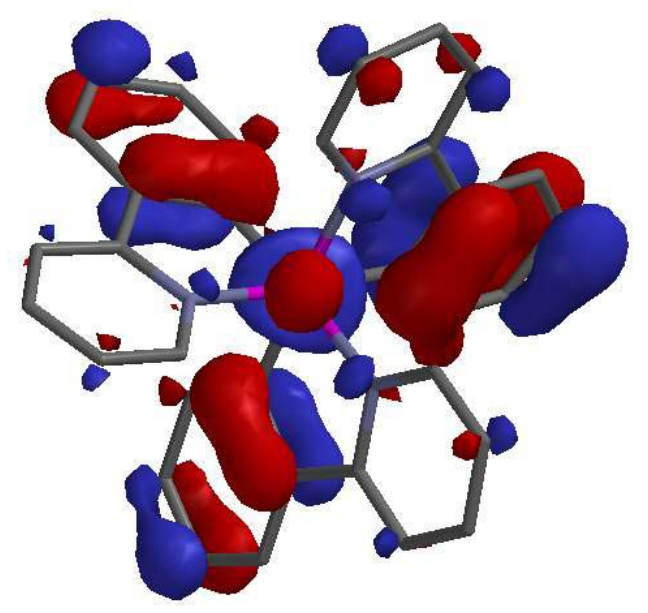




\section{Gapma}

HOMO (-4.99 eV)

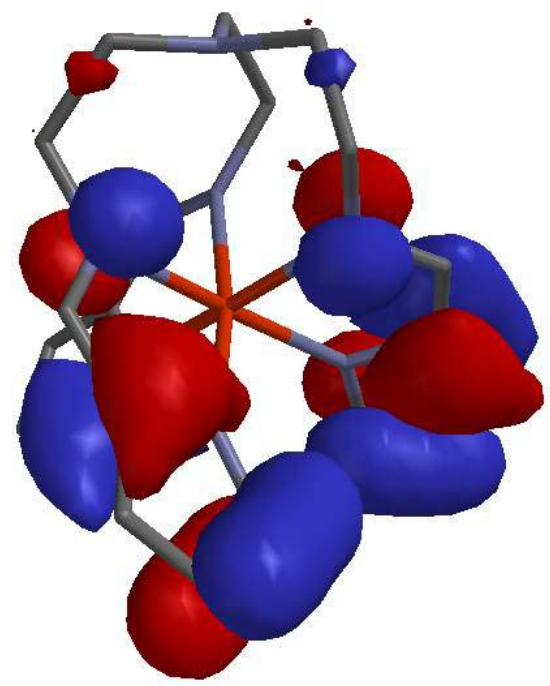

LUMO (-0.75 eV)

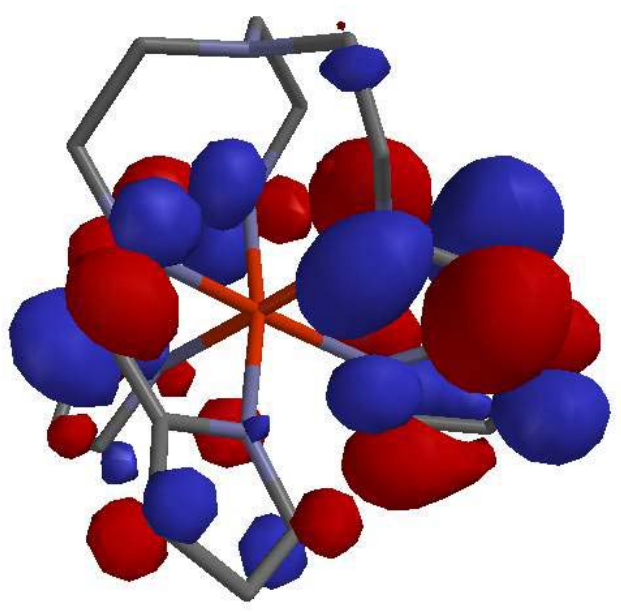


Figure S 4. Ortep plots for $f a c-\operatorname{Co}(p p z)_{3}$, Gapma and $\operatorname{Co}\left(p p z_{2}\right) \mathrm{Cl}_{2}$.

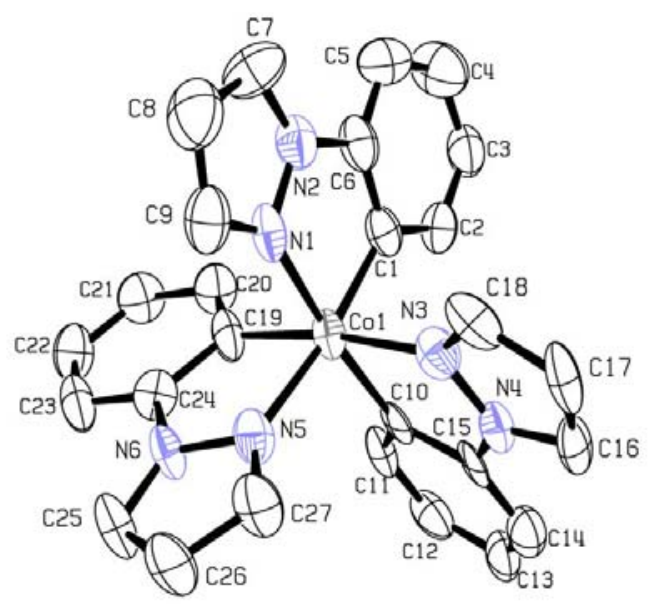

$f a c-\operatorname{Co}(p p z)_{3}$

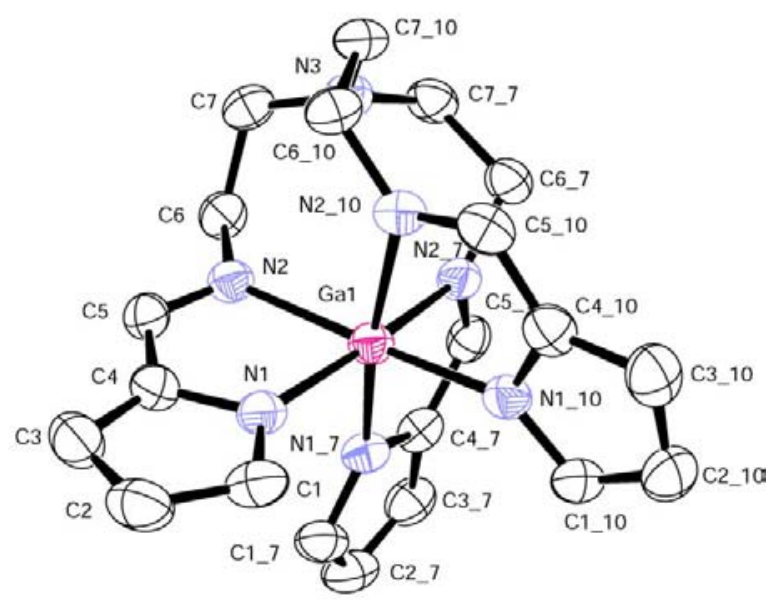

Gapma

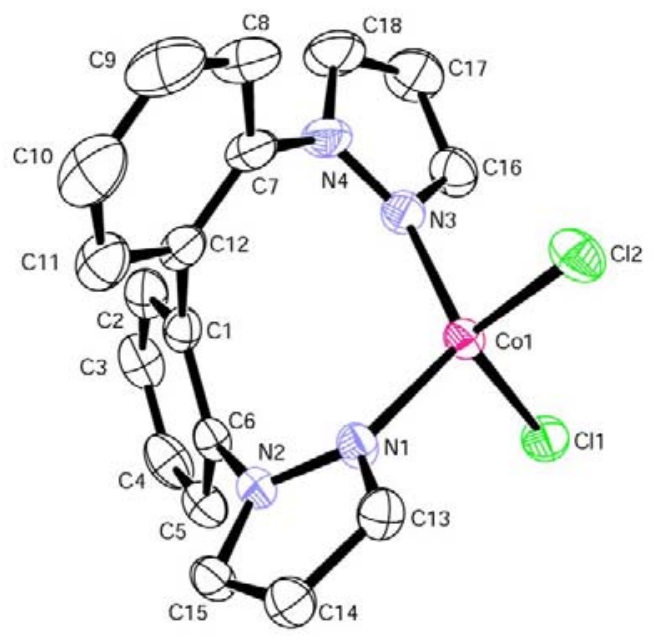

\section{$\operatorname{Co}\left(p p z_{2}\right) \mathrm{Cl}_{2}$}


Table S 1. Atomic coordinates ( x 104) and equivalent isotropic displacement parameters $(\AA 2 \mathrm{x}$ 103)for $\mathrm{fac}-\mathrm{Co}(\mathrm{ppz}) 3.0 .5 \mathrm{CH} 2 \mathrm{Cl2}$. U(eq) is defined as one third of the trace of the orthogonalized Uij tensor.

\begin{tabular}{|c|c|c|c|c|}
\hline & $\mathrm{x}$ & $\mathrm{y}$ & $\mathrm{z}$ & $\mathrm{U}(\mathrm{eq})$ \\
\hline $\operatorname{Co}(1)$ & $7763(1)$ & $4601(1)$ & $914(1)$ & $17(1)$ \\
\hline $\operatorname{Co}(2)$ & $7797(1)$ & $9625(1)$ & $914(1)$ & $16(1)$ \\
\hline $\mathrm{N}(1)$ & $7858(3)$ & $5290(3)$ & $1646(2)$ & $20(1)$ \\
\hline $\mathrm{N}(2)$ & $7330(3)$ & $4951(3)$ & $2043(2)$ & $22(1)$ \\
\hline $\mathrm{N}(3)$ & $6660(3)$ & $5322(3)$ & $509(2)$ & $20(1)$ \\
\hline $\mathrm{N}(4)$ & $6376(3)$ & $5119(3)$ & $-72(2)$ & $18(1)$ \\
\hline $\mathrm{N}(5)$ & $8728(3)$ & $5356(3)$ & $623(2)$ & $19(1)$ \\
\hline $\mathrm{N}(6)$ & $9697(3)$ & $5113(3)$ & $797(2)$ & $19(1)$ \\
\hline $\mathrm{N}(7)$ & $6628(3)$ & $10348(3)$ & $621(2)$ & $17(1)$ \\
\hline $\mathrm{N}(8)$ & $5770(3)$ & $10063(3)$ & $785(2)$ & $18(1)$ \\
\hline $\mathrm{N}(9)$ & $8145(3)$ & $10331(3)$ & $1636(2)$ & $21(1)$ \\
\hline $\mathrm{N}(10)$ & $8983(4)$ & $10067(3)$ & 1999(2) & $24(1)$ \\
\hline $\mathrm{N}(11)$ & $8623(3)$ & 10354(3) & $496(2)$ & $19(1)$ \\
\hline $\mathrm{N}(12)$ & $8574(3)$ & $10132(3)$ & $-76(2)$ & $18(1)$ \\
\hline $\mathrm{Cl}(1)$ & $3540(1)$ & 3953(1) & $1743(1)$ & $41(1)$ \\
\hline $\mathrm{Cl}(2)$ & $4442(2)$ & $5447(1)$ & $2425(1)$ & $51(1)$ \\
\hline $\mathrm{C}(1)$ & $6937(4)$ & $3878(4)$ & $1296(2)$ & $20(1)$ \\
\hline$C(2)$ & $6437(4)$ & $3101(4)$ & $1125(3)$ & $20(1)$ \\
\hline$C(3)$ & $5896(4)$ & $2655(4)$ & $1480(3)$ & $25(2)$ \\
\hline$C(4)$ & $5843(5)$ & $2955(4)$ & $2035(3)$ & $32(2)$ \\
\hline$C(5)$ & $6318(5)$ & $3720(4)$ & $2240(3)$ & $31(2)$ \\
\hline$C(6)$ & $6830(4)$ & $4157(4)$ & 1861(3) & $20(1)$ \\
\hline$C(7)$ & $7401(5)$ & $5458(4)$ & $2523(3)$ & $28(2)$ \\
\hline$C(8)$ & $7975(5)$ & $6146(4)$ & $2440(3)$ & $34(2)$ \\
\hline $\mathrm{C}(9)$ & $8250(4)$ & $6018(4)$ & $1887(3)$ & $25(1)$ \\
\hline$C(10)$ & $7510(4)$ & $3974(4)$ & $179(2)$ & $17(1)$ \\
\hline $\mathrm{C}(11)$ & $7911(4)$ & $3197(4)$ & $12(3)$ & $21(1)$ \\
\hline$C(12)$ & $7670(4)$ & 2881(4) & $-559(3)$ & $24(1)$ \\
\hline$C(13)$ & $7006(4)$ & $3326(4)$ & $-993(3)$ & $22(1)$ \\
\hline
\end{tabular}




\begin{tabular}{|c|c|c|c|c|}
\hline$C(14)$ & $6574(4)$ & $4080(4)$ & $-840(3)$ & $23(1)$ \\
\hline$C(15)$ & $6829(4)$ & $4369(4)$ & $-264(2)$ & $18(1)$ \\
\hline$C(16)$ & $5650(4)$ & $5673(4)$ & $-343(3)$ & $20(1)$ \\
\hline$C(17)$ & $5490(4)$ & $6248(4)$ & $77(3)$ & $23(1)$ \\
\hline$C(18)$ & $6135(4)$ & $6014(4)$ & $601(3)$ & $21(1)$ \\
\hline$C(19)$ & $8939(4)$ & $3942(4)$ & $1222(3)$ & $20(1)$ \\
\hline$C(20)$ & $9066(4)$ & $3157(4)$ & $1528(3)$ & $22(1)$ \\
\hline $\mathrm{C}(21)$ & $10007(4)$ & $2812(4)$ & $1735(3)$ & $24(1)$ \\
\hline$C(22)$ & $10864(4)$ & $3228(4)$ & 1641(3) & $25(2)$ \\
\hline $\mathrm{C}(23)$ & $10776(4)$ & $4009(4)$ & $1332(3)$ & $25(2)$ \\
\hline$C(24)$ & $9827(4)$ & $4332(4)$ & $1130(2)$ & $17(1)$ \\
\hline$C(25)$ & $10326(4)$ & $5645(4)$ & $578(3)$ & $24(1)$ \\
\hline$C(26)$ & $9741(4)$ & $6255(4)$ & $252(3)$ & $25(2)$ \\
\hline $\mathrm{C}(27)$ & $8762(4)$ & $6061(4)$ & 291(3) & $24(1)$ \\
\hline $\mathrm{C}(28)$ & $6842(4)$ & $8940(4)$ & $1229(2)$ & $18(1)$ \\
\hline C(29) & $6944(5)$ & $8168(4)$ & $1549(3)$ & $23(1)$ \\
\hline$C(30)$ & $6135(5)$ & $7765(4)$ & $1720(3)$ & $27(2)$ \\
\hline $\mathrm{C}(31)$ & $5183(5)$ & $8118(4)$ & $1580(3)$ & $29(2)$ \\
\hline$C(32)$ & $5036(4)$ & $8877(4)$ & $1272(3)$ & $25(1)$ \\
\hline C(33) & $5868(4)$ & $9284(4)$ & $1110(2)$ & $21(1)$ \\
\hline$C(34)$ & 4997(4) & $10597(4)$ & $579(3)$ & $25(2)$ \\
\hline$C(35)$ & $5359(4)$ & $11236(4)$ & $276(3)$ & $21(1)$ \\
\hline$C(36)$ & $6382(4)$ & $11061(4)$ & $308(3)$ & $21(1)$ \\
\hline$C(37)$ & $8930(4)$ & 8961(4) & $1283(2)$ & $19(1)$ \\
\hline$C(38)$ & $9354(4)$ & $8201(4)$ & $1116(3)$ & $20(1)$ \\
\hline C(39) & $10263(4)$ & $7886(4)$ & $1419(3)$ & $25(2)$ \\
\hline$C(40)$ & $10780(5)$ & $8325(4)$ & $1908(3)$ & $31(2)$ \\
\hline$C(41)$ & $10372(5)$ & $9064(4)$ & $2109(3)$ & $28(2)$ \\
\hline$C(42)$ & $9461(4)$ & $9319(4)$ & 1801(3) & $21(1)$ \\
\hline$C(43)$ & $9159(5)$ & $10560(4)$ & $2490(2)$ & $21(1)$ \\
\hline$C(44)$ & $8449(5)$ & $11175(4)$ & $2445(3)$ & $29(2)$ \\
\hline$C(45)$ & $7815(4)$ & $11014(4)$ & 1903(3) & $24(1)$ \\
\hline$C(46)$ & $7610(4)$ & $8978(4)$ & $189(2)$ & $16(1)$ \\
\hline$C(47)$ & $7112(4)$ & $8200(4)$ & $24(2)$ & $18(1)$ \\
\hline$C(48)$ & $7000(4)$ & $7881(4)$ & $-556(3)$ & $22(1)$ \\
\hline$C(49)$ & $7404(4)$ & $8317(4)$ & $-986(3)$ & $22(1)$ \\
\hline
\end{tabular}




\begin{tabular}{lrrrr}
$\mathrm{C}(50)$ & $7929(4)$ & $9073(4)$ & $-833(3)$ & $20(1)$ \\
$\mathrm{C}(51)$ & $8018(4)$ & $9382(4)$ & $-262(3)$ & $19(1)$ \\
$\mathrm{C}(52)$ & $9141(4)$ & $10673(4)$ & $-352(3)$ & $23(1)$ \\
$\mathrm{C}(53)$ & $9554(4)$ & $11262(4)$ & $68(3)$ & $24(1)$ \\
$\mathrm{C}(54)$ & $9217(4)$ & $11048(4)$ & $597(3)$ & $22(1)$ \\
$\mathrm{C}(55)$ & $3471(6)$ & $4664(5)$ & $2321(3)$ & $49(2)$ \\
\hline
\end{tabular}

Table S 2. Bond lengths $[\AA]$ and angles $\left[{ }^{\circ}\right]$ for $f a c-C o(p p z) 3.0 .5 C H 2 C l 2$.

\begin{tabular}{ll}
\hline $\mathrm{Co}(1)-\mathrm{C}(1)$ & $1.913(6)$ \\
$\mathrm{Co}(1)-\mathrm{C}(19)$ & $1.924(6)$ \\
$\mathrm{Co}(1)-\mathrm{C}(10)$ & $1.925(6)$ \\
$\mathrm{Co}(1)-\mathrm{N}(3)$ & $1.966(5)$ \\
$\mathrm{Co}(1)-\mathrm{N}(5)$ & $1.968(5)$ \\
$\mathrm{Co}(1)-\mathrm{N}(1)$ & $1.979(5)$ \\
$\mathrm{Co}(2)-\mathrm{C}(37)$ & $1.919(6)$ \\
$\mathrm{Co}(2)-\mathrm{C}(28)$ & $1.921(6)$ \\
$\mathrm{Co}(2)-\mathrm{C}(46)$ & $1.922(6)$ \\
$\mathrm{Co}(2)-\mathrm{N}(7)$ & $1.965(5)$ \\
$\mathrm{Co}(2)-\mathrm{N}(11)$ & $1.968(5)$ \\
$\mathrm{Co}(2)-\mathrm{N}(9)$ & $1.974(5)$ \\
$\mathrm{N}(1)-\mathrm{C}(9)$ & $1.328(7)$ \\
$\mathrm{N}(1)-\mathrm{N}(2)$ & $1.366(7)$ \\
$\mathrm{N}(2)-\mathrm{C}(7)$ & $1.344(7)$ \\
$\mathrm{N}(2)-\mathrm{C}(6)$ & $1.436(7)$ \\
$\mathrm{N}(3)-\mathrm{C}(18)$ & $1.330(7)$ \\
$\mathrm{N}(3)-\mathrm{N}(4)$ & $1.356(6)$ \\
$\mathrm{N}(4)-\mathrm{C}(16)$ & $1.373(7)$ \\
$\mathrm{N}(4)-\mathrm{C}(15)$ & $1.427(7)$ \\
$\mathrm{N}(5)-\mathrm{C}(27)$ & $1.341(7)$ \\
$\mathrm{N}(5)-\mathrm{N}(6)$ & $1.361(6)$ \\
& \\
& \\
&
\end{tabular}




\begin{tabular}{|c|c|}
\hline $\mathrm{N}(6)-\mathrm{C}(25)$ & $1.352(7)$ \\
\hline $\mathrm{N}(6)-\mathrm{C}(24)$ & $1.429(7)$ \\
\hline $\mathrm{N}(7)-\mathrm{C}(36)$ & $1.330(7)$ \\
\hline $\mathrm{N}(7)-\mathrm{N}(8)$ & $1.365(6)$ \\
\hline N(8)-C(34) & $1.357(7)$ \\
\hline N(8)-C(33) & $1.416(8)$ \\
\hline $\mathrm{N}(9)-\mathrm{C}(45)$ & $1.344(7)$ \\
\hline $\mathrm{N}(9)-\mathrm{N}(10)$ & $1.347(6)$ \\
\hline $\mathrm{N}(10)-\mathrm{C}(43)$ & $1.349(7)$ \\
\hline $\mathrm{N}(10)-\mathrm{C}(42)$ & $1.446(8)$ \\
\hline $\mathrm{N}(11)-\mathrm{C}(54)$ & $1.343(7)$ \\
\hline $\mathrm{N}(11)-\mathrm{N}(12)$ & $1.348(6)$ \\
\hline $\mathrm{N}(12)-\mathrm{C}(52)$ & $1.373(7)$ \\
\hline $\mathrm{N}(12)-\mathrm{C}(51)$ & $1.414(7)$ \\
\hline $\mathrm{Cl}(1)-\mathrm{C}(55)$ & $1.742(8)$ \\
\hline $\mathrm{Cl}(2)-\mathrm{C}(55)$ & $1.782(8)$ \\
\hline$C(1)-C(6)$ & $1.399(8)$ \\
\hline $\mathrm{C}(1)-\mathrm{C}(2)$ & $1.409(8)$ \\
\hline$C(2)-C(3)$ & $1.380(8)$ \\
\hline$C(3)-C(4)$ & $1.372(9)$ \\
\hline$C(4)-C(5)$ & $1.395(9)$ \\
\hline$C(5)-C(6)$ & $1.389(8)$ \\
\hline$C(7)-C(8)$ & $1.358(9)$ \\
\hline $\mathrm{C}(8)-\mathrm{C}(9)$ & $1.403(9)$ \\
\hline$C(10)-C(15)$ & $1.389(8)$ \\
\hline$C(10)-C(11)$ & $1.408(8)$ \\
\hline $\mathrm{C}(11)-\mathrm{C}(12)$ & $1.382(8)$ \\
\hline$C(12)-C(13)$ & $1.399(8)$ \\
\hline $\mathrm{C}(13)-\mathrm{C}(14)$ & $1.385(8)$ \\
\hline$C(14)-C(15)$ & $1.379(8)$ \\
\hline$C(16)-C(17)$ & $1.362(8)$ \\
\hline$C(17)-C(18)$ & $1.403(8)$ \\
\hline$C(19)-C(24)$ & $1.403(8)$ \\
\hline$C(19)-C(20)$ & $1.404(8)$ \\
\hline $\mathrm{C}(20)-\mathrm{C}(21)$ & $1.389(8)$ \\
\hline $\mathrm{C}(21)-\mathrm{C}(22)$ & $1.385(8)$ \\
\hline
\end{tabular}




\begin{tabular}{|c|c|}
\hline$C(22)-C(23)$ & $1.401(9)$ \\
\hline$C(23)-C(24)$ & $1.384(8)$ \\
\hline$C(25)-C(26)$ & $1.370(8)$ \\
\hline$C(26)-C(27)$ & $1.385(8)$ \\
\hline $\mathrm{C}(28)-\mathrm{C}(29)$ & $1.402(8)$ \\
\hline$C(28)-C(33)$ & $1.410(8)$ \\
\hline$C(29)-C(30)$ & $1.385(8)$ \\
\hline $\mathrm{C}(30)-\mathrm{C}(31)$ & $1.389(9)$ \\
\hline $\mathrm{C}(31)-\mathrm{C}(32)$ & $1.374(9)$ \\
\hline $\mathrm{C}(32)-\mathrm{C}(33)$ & $1.404(8)$ \\
\hline$C(34)-C(35)$ & $1.357(9)$ \\
\hline$C(35)-C(36)$ & $1.408(8)$ \\
\hline $\mathrm{C}(37)-\mathrm{C}(42)$ & $1.391(8)$ \\
\hline $\mathrm{C}(37)-\mathrm{C}(38)$ & $1.400(8)$ \\
\hline $\mathrm{C}(38)-\mathrm{C}(39)$ & $1.393(8)$ \\
\hline$C(39)-C(40)$ & $1.390(9)$ \\
\hline $\mathrm{C}(40)-\mathrm{C}(41)$ & $1.391(9)$ \\
\hline$C(41)-C(42)$ & $1.369(8)$ \\
\hline$C(43)-C(44)$ & $1.350(8)$ \\
\hline$C(44)-C(45)$ & $1.401(8)$ \\
\hline$C(46)-C(47)$ & $1.406(8)$ \\
\hline$C(46)-C(51)$ & $1.409(8)$ \\
\hline$C(47)-C(48)$ & $1.404(8)$ \\
\hline$C(48)-C(49)$ & $1.391(8)$ \\
\hline$C(49)-C(50)$ & $1.387(8)$ \\
\hline$C(50)-C(51)$ & $1.380(8)$ \\
\hline$C(52)-C(53)$ & $1.375(8)$ \\
\hline$C(53)-C(54)$ & $1.414(8)$ \\
\hline $\mathrm{C}(1)-\mathrm{Co}(1)-\mathrm{C}(19)$ & $92.1(2)$ \\
\hline $\mathrm{C}(1)-\mathrm{Co}(1)-\mathrm{C}(10)$ & $94.5(2)$ \\
\hline$C(19)-\operatorname{Co}(1)-C(10)$ & $93.2(2)$ \\
\hline $\mathrm{C}(1)-\mathrm{Co}(1)-\mathrm{N}(3)$ & $95.2(2)$ \\
\hline $\mathrm{C}(19)-\mathrm{Co}(1)-\mathrm{N}(3)$ & $171.9(2)$ \\
\hline $\mathrm{C}(10)-\mathrm{Co}(1)-\mathrm{N}(3)$ & $82.9(2)$ \\
\hline $\mathrm{C}(1)-\mathrm{Co}(1)-\mathrm{N}(5)$ & $172.5(2)$ \\
\hline
\end{tabular}




\begin{tabular}{|c|c|}
\hline $\mathrm{C}(19)-\mathrm{Co}(1)-\mathrm{N}(5)$ & $83.1(2)$ \\
\hline $\mathrm{C}(10)-\mathrm{Co}(1)-\mathrm{N}(5)$ & $91.6(2)$ \\
\hline $\mathrm{N}(3)-\mathrm{Co}(1)-\mathrm{N}(5)$ & $89.90(19)$ \\
\hline $\mathrm{C}(1)-\mathrm{Co}(1)-\mathrm{N}(1)$ & $83.2(2)$ \\
\hline $\mathrm{C}(19)-\mathrm{Co}(1)-\mathrm{N}(1)$ & $93.2(2)$ \\
\hline $\mathrm{C}(10)-\mathrm{Co}(1)-\mathrm{N}(1)$ & $173.3(2)$ \\
\hline $\mathrm{N}(3)-\mathrm{Co}(1)-\mathrm{N}(1)$ & $91.03(19)$ \\
\hline $\mathrm{N}(5)-\mathrm{Co}(1)-\mathrm{N}(1)$ & $91.2(2)$ \\
\hline $\mathrm{C}(37)-\mathrm{Co}(2)-\mathrm{C}(28)$ & $94.6(2)$ \\
\hline$C(37)-\operatorname{Co}(2)-C(46)$ & $93.9(2)$ \\
\hline $\mathrm{C}(28)-\mathrm{Co}(2)-\mathrm{C}(46)$ & $92.5(2)$ \\
\hline $\mathrm{C}(37)-\mathrm{Co}(2)-\mathrm{N}(7)$ & $173.9(2)$ \\
\hline $\mathrm{C}(28)-\mathrm{Co}(2)-\mathrm{N}(7)$ & $83.4(2)$ \\
\hline $\mathrm{C}(46)-\mathrm{Co}(2)-\mathrm{N}(7)$ & $91.9(2)$ \\
\hline $\mathrm{C}(37)-\mathrm{Co}(2)-\mathrm{N}(11)$ & $92.3(2)$ \\
\hline $\mathrm{C}(28)-\mathrm{Co}(2)-\mathrm{N}(11)$ & $171.9(2)$ \\
\hline $\mathrm{C}(46)-\mathrm{Co}(2)-\mathrm{N}(11)$ & $82.9(2)$ \\
\hline $\mathrm{N}(7)-\mathrm{Co}(2)-\mathrm{N}(11)$ & $90.10(19)$ \\
\hline $\mathrm{C}(37)-\mathrm{Co}(2)-\mathrm{N}(9)$ & $83.0(2)$ \\
\hline $\mathrm{C}(28)-\mathrm{Co}(2)-\mathrm{N}(9)$ & $93.9(2)$ \\
\hline $\mathrm{C}(46)-\mathrm{Co}(2)-\mathrm{N}(9)$ & $173.1(2)$ \\
\hline $\mathrm{N}(7)-\mathrm{Co}(2)-\mathrm{N}(9)$ & $91.37(19)$ \\
\hline $\mathrm{N}(11)-\mathrm{Co}(2)-\mathrm{N}(9)$ & $91.0(2)$ \\
\hline $\mathrm{C}(9)-\mathrm{N}(1)-\mathrm{N}(2)$ & $105.7(5)$ \\
\hline $\mathrm{C}(9)-\mathrm{N}(1)-\mathrm{Co}(1)$ & $140.8(4)$ \\
\hline $\mathrm{N}(2)-\mathrm{N}(1)-\mathrm{Co}(1)$ & $113.4(4)$ \\
\hline $\mathrm{C}(7)-\mathrm{N}(2)-\mathrm{N}(1)$ & $110.6(5)$ \\
\hline $\mathrm{C}(7)-\mathrm{N}(2)-\mathrm{C}(6)$ & $134.7(5)$ \\
\hline $\mathrm{N}(1)-\mathrm{N}(2)-\mathrm{C}(6)$ & $114.6(5)$ \\
\hline $\mathrm{C}(18)-\mathrm{N}(3)-\mathrm{N}(4)$ & $106.3(5)$ \\
\hline $\mathrm{C}(18)-\mathrm{N}(3)-\mathrm{Co}(1)$ & $140.6(4)$ \\
\hline $\mathrm{N}(4)-\mathrm{N}(3)-\mathrm{Co}(1)$ & $112.8(3)$ \\
\hline $\mathrm{N}(3)-\mathrm{N}(4)-\mathrm{C}(16)$ & $110.7(5)$ \\
\hline $\mathrm{N}(3)-\mathrm{N}(4)-\mathrm{C}(15)$ & $115.7(4)$ \\
\hline$C(16)-N(4)-C(15)$ & $133.5(5)$ \\
\hline $\mathrm{C}(27)-\mathrm{N}(5)-\mathrm{N}(6)$ & $105.2(5)$ \\
\hline
\end{tabular}




\begin{tabular}{|c|c|}
\hline $\mathrm{C}(27)-\mathrm{N}(5)-\mathrm{Co}(1)$ & $140.8(4)$ \\
\hline $\mathrm{N}(6)-\mathrm{N}(5)-\mathrm{Co}(1)$ & $114.0(4)$ \\
\hline $\mathrm{C}(25)-\mathrm{N}(6)-\mathrm{N}(5)$ & $111.5(5)$ \\
\hline $\mathrm{C}(25)-\mathrm{N}(6)-\mathrm{C}(24)$ & $134.0(5)$ \\
\hline $\mathrm{N}(5)-\mathrm{N}(6)-\mathrm{C}(24)$ & $114.3(4)$ \\
\hline $\mathrm{C}(36)-\mathrm{N}(7)-\mathrm{N}(8)$ & $106.1(5)$ \\
\hline $\mathrm{C}(36)-\mathrm{N}(7)-\mathrm{Co}(2)$ & $140.4(4)$ \\
\hline $\mathrm{N}(8)-\mathrm{N}(7)-\mathrm{Co}(2)$ & $113.5(4)$ \\
\hline $\mathrm{C}(34)-\mathrm{N}(8)-\mathrm{N}(7)$ & $110.7(5)$ \\
\hline $\mathrm{C}(34)-\mathrm{N}(8)-\mathrm{C}(33)$ & $134.4(5)$ \\
\hline N(7)-N(8)-C(33) & $114.9(4)$ \\
\hline $\mathrm{C}(45)-\mathrm{N}(9)-\mathrm{N}(10)$ & $106.0(5)$ \\
\hline $\mathrm{C}(45)-\mathrm{N}(9)-\mathrm{Co}(2)$ & $140.7(4)$ \\
\hline $\mathrm{N}(10)-\mathrm{N}(9)-\mathrm{Co}(2)$ & $113.3(4)$ \\
\hline $\mathrm{N}(9)-\mathrm{N}(10)-\mathrm{C}(43)$ & $110.1(5)$ \\
\hline $\mathrm{N}(9)-\mathrm{N}(10)-\mathrm{C}(42)$ & $115.5(5)$ \\
\hline $\mathrm{C}(43)-\mathrm{N}(10)-\mathrm{C}(42)$ & $134.4(5)$ \\
\hline $\mathrm{C}(54)-\mathrm{N}(11)-\mathrm{N}(12)$ & $107.2(5)$ \\
\hline $\mathrm{C}(54)-\mathrm{N}(11)-\mathrm{Co}(2)$ & $139.4(4)$ \\
\hline $\mathrm{N}(12)-\mathrm{N}(11)-\mathrm{Co}(2)$ & $113.3(3)$ \\
\hline $\mathrm{N}(11)-\mathrm{N}(12)-\mathrm{C}(52)$ & $111.1(5)$ \\
\hline $\mathrm{N}(11)-\mathrm{N}(12)-\mathrm{C}(51)$ & $115.8(5)$ \\
\hline $\mathrm{C}(52)-\mathrm{N}(12)-\mathrm{C}(51)$ & $133.0(5)$ \\
\hline$C(6)-C(1)-C(2)$ & $113.4(5)$ \\
\hline $\mathrm{C}(6)-\mathrm{C}(1)-\mathrm{Co}(1)$ & $114.3(4)$ \\
\hline$C(2)-C(1)-C o(1)$ & $132.2(4)$ \\
\hline $\mathrm{C}(3)-\mathrm{C}(2)-\mathrm{C}(1)$ & $123.3(6)$ \\
\hline $\mathrm{C}(4)-\mathrm{C}(3)-\mathrm{C}(2)$ & $120.1(6)$ \\
\hline$C(3)-C(4)-C(5)$ & $120.3(6)$ \\
\hline$C(6)-C(5)-C(4)$ & $117.4(6)$ \\
\hline$C(5)-C(6)-C(1)$ & $125.4(6)$ \\
\hline $\mathrm{C}(5)-\mathrm{C}(6)-\mathrm{N}(2)$ & $120.2(5)$ \\
\hline $\mathrm{C}(1)-\mathrm{C}(6)-\mathrm{N}(2)$ & $114.4(5)$ \\
\hline $\mathrm{N}(2)-\mathrm{C}(7)-\mathrm{C}(8)$ & $107.8(6)$ \\
\hline $\mathrm{C}(7)-\mathrm{C}(8)-\mathrm{C}(9)$ & $105.6(6)$ \\
\hline $\mathrm{N}(1)-\mathrm{C}(9)-\mathrm{C}(8)$ & $110.2(6)$ \\
\hline
\end{tabular}




$\begin{array}{ll}\mathrm{C}(15)-\mathrm{C}(10)-\mathrm{C}(11) & 114.9(5) \\ \mathrm{C}(15)-\mathrm{C}(10)-\mathrm{C}(1) & 114.0(4) \\ \mathrm{C}(11)-\mathrm{C}(10)-\mathrm{Co}(1) & 131.1(4) \\ \mathrm{C}(12)-\mathrm{C}(11)-\mathrm{C}(10) & 121.8(6) \\ \mathrm{C}(11)-\mathrm{C}(12)-\mathrm{C}(13) & 120.6(6) \\ \mathrm{C}(14)-\mathrm{C}(13)-\mathrm{C}(12) & 119.2(6) \\ \mathrm{C}(15)-\mathrm{C}(14)-\mathrm{C}(13) & 118.3(6) \\ \mathrm{C}(14)-\mathrm{C}(15)-\mathrm{C}(10) & 125.1(6) \\ \mathrm{C}(14)-\mathrm{C}(15)-\mathrm{N}(4) & 121.1(5) \\ \mathrm{C}(10)-\mathrm{C}(15)-\mathrm{N}(4) & 113.8(5) \\ \mathrm{C}(17)-\mathrm{C}(16)-\mathrm{N}(4) & 106.6(5) \\ \mathrm{C}(16)-\mathrm{C}(17)-\mathrm{C}(18) & 106.4(5) \\ \mathrm{N}(3)-\mathrm{C}(18)-\mathrm{C}(17) & 110.1(5) \\ \mathrm{C}(24)-\mathrm{C}(19)-\mathrm{C}(20) & 114.9(5) \\ \mathrm{C}(24)-\mathrm{C}(19)-\mathrm{Co}(1) & 113.3(4) \\ \mathrm{C}(20)-\mathrm{C}(19)-\mathrm{Co}(1) & 131.7(4) \\ \mathrm{C}(21)-\mathrm{C}(20)-\mathrm{C}(19) & 121.9(6) \\ \mathrm{C}(22)-\mathrm{C}(21)-\mathrm{C}(20) & 121.1(6) \\ \mathrm{C}(21)-\mathrm{C}(22)-\mathrm{C}(23) & 119.1(5) \\ \mathrm{C}(24)-\mathrm{C}(23)-\mathrm{C}(22) & 118.3(6) \\ \mathrm{C}(23)-\mathrm{C}(24)-\mathrm{C}(19) & 124.6(6) \\ \mathrm{C}(23)-\mathrm{C}(24)-\mathrm{N}(6) & 120.3(5) \\ \mathrm{C}(19)-\mathrm{C}(24)-\mathrm{N}(6) & 115.0(5) \\ \mathrm{N}(6)-\mathrm{C}(25)-\mathrm{C}(26) & 106.5(5) \\ \mathrm{C}(25)-\mathrm{C}(26)-\mathrm{C}(27) & 106.5(5) \\ \mathrm{N}(5)-\mathrm{C}(27)-\mathrm{C}(26) & 110.4(5) \\ \mathrm{C}(29)-\mathrm{C}(28)-\mathrm{C}(33) & 115.3(5) \\ \mathrm{C}(29)-\mathrm{C}(28)-\mathrm{Co}(2) & 131.6(4) \\ \mathrm{C}(33)-\mathrm{C}(28)-\mathrm{Co}(2) & 113.2(4) \\ \mathrm{C}(30)-\mathrm{C}(29)-\mathrm{C}(28) & 121.9(6) \\ \mathrm{C}(29)-\mathrm{C}(30)-\mathrm{C}(31) & 120.8(6) \\ \mathrm{C}(32)-\mathrm{C}(31)-\mathrm{C}(30) & 120.1(6) \\ & 118.4(6) \\ \mathrm{C}(32)-\mathrm{C}(33) & 123.5(6) \\ \mathrm{C}(28) & 121.5(5) \\ & \end{array}$




\begin{tabular}{ll}
$\mathrm{C}(35)-\mathrm{C}(34)-\mathrm{N}(8)$ & $107.2(5)$ \\
$\mathrm{C}(34)-\mathrm{C}(35)-\mathrm{C}(36)$ & $106.2(5)$ \\
$\mathrm{N}(7)-\mathrm{C}(36)-\mathrm{C}(35)$ & $109.8(5)$ \\
$\mathrm{C}(42)-\mathrm{C}(37)-\mathrm{C}(38)$ & $113.3(5)$ \\
$\mathrm{C}(42)-\mathrm{C}(37)-\mathrm{Co}(2)$ & $114.3(4)$ \\
$\mathrm{C}(38)-\mathrm{C}(37)-\mathrm{Co}(2)$ & $132.3(4)$ \\
$\mathrm{C}(39)-\mathrm{C}(38)-\mathrm{C}(37)$ & $122.3(6)$ \\
$\mathrm{C}(40)-\mathrm{C}(39)-\mathrm{C}(38)$ & $120.1(6)$ \\
$\mathrm{C}(39)-\mathrm{C}(40)-\mathrm{C}(41)$ & $120.0(6)$ \\
$\mathrm{C}(42)-\mathrm{C}(41)-\mathrm{C}(40)$ & $116.6(6)$ \\
$\mathrm{C}(41)-\mathrm{C}(42)-\mathrm{C}(37)$ & $127.3(6)$ \\
$\mathrm{C}(41)-\mathrm{C}(42)-\mathrm{N}(10)$ & $119.2(5)$ \\
$\mathrm{C}(37)-\mathrm{C}(42)-\mathrm{N}(10)$ & $113.4(5)$ \\
$\mathrm{N}(10)-\mathrm{C}(43)-\mathrm{C}(44)$ & $108.9(5)$ \\
$\mathrm{C}(43)-\mathrm{C}(44)-\mathrm{C}(45)$ & $104.9(5)$ \\
$\mathrm{N}(9)-\mathrm{C}(45)-\mathrm{C}(44)$ & $110.0(6)$ \\
$\mathrm{C}(47)-\mathrm{C}(46)-\mathrm{C}(51)$ & $114.9(5)$ \\
$\mathrm{C}(47)-\mathrm{C}(46)-\mathrm{Co}(2)$ & $131.7(4)$ \\
$\mathrm{C}(51)-\mathrm{C}(46)-\mathrm{Co}(2)$ & $113.3(4)$ \\
$\mathrm{C}(48)-\mathrm{C}(47)-\mathrm{C}(46)$ & $121.6(5)$ \\
$\mathrm{C}(49)-\mathrm{C}(48)-\mathrm{C}(47)$ & $120.9(6)$ \\
$\mathrm{C}(50)-\mathrm{C}(49)-\mathrm{C}(48)$ & $119.0(6)$ \\
$\mathrm{C}(51)-\mathrm{C}(50)-\mathrm{C}(49)$ & $119.2(6)$ \\
$\mathrm{C}(50)-\mathrm{C}(51)-\mathrm{C}(46)$ & $124.4(5)$ \\
$\mathrm{C}(50)-\mathrm{C}(51)-\mathrm{N}(12)$ & $121.6(5)$ \\
$\mathrm{C}(46)-\mathrm{C}(51)-\mathrm{N}(12)$ & $114.0(5)$ \\
$\mathrm{N}(12)-\mathrm{C}(52)-\mathrm{C}(53)$ & $106.1(5)$ \\
$\mathrm{C}(52)-\mathrm{C}(53)-\mathrm{C}(54)$ & $106.8(5)$ \\
$\mathrm{N}(11)-\mathrm{C}(54)-\mathrm{C}(53)$ & $108.7(5)$ \\
$\mathrm{Cl}(1)-\mathrm{C}(55)-\mathrm{Cl}(2)$ & $113.3(4)$ \\
\hline
\end{tabular}

Table S 3. Anisotropic displacement parameters ( $\AA 2 \mathrm{x} 103)$ for $f a c-C o(p p z) 3.0 .5 C H 2 C l 2$. The anisotropic displacement factor exponent takes the form: $-2 \quad 2\left[\mathrm{~h} 2 \mathrm{a} * 2 \mathrm{U} 11+\ldots+2 \mathrm{~h} \mathrm{k} \mathrm{a}^{*} \mathrm{~b}^{*}\right.$ 
U12 ].

\begin{tabular}{lcccccc}
\hline & $\mathrm{U} 11$ & $\mathrm{U} 22$ & $\mathrm{U} 33$ & $\mathrm{U}^{23}$ & $\mathrm{U} 13$ & $\mathrm{U} 12$ \\
\hline $\mathrm{Co}(1)$ & $10(1)$ & $15(1)$ & $23(1)$ & $0(1)$ & $0(1)$ & $0(1)$ \\
$\mathrm{Co}(2)$ & $12(1)$ & $15(1)$ & $21(1)$ & $0(1)$ & $1(1)$ & $0(1)$ \\
$\mathrm{N}(1)$ & $9(2)$ & $21(3)$ & $29(3)$ & $0(2)$ & $-2(2)$ & $1(2)$ \\
$\mathrm{N}(2)$ & $16(3)$ & $23(3)$ & $26(3)$ & $-3(2)$ & $1(2)$ & $-2(2)$ \\
$\mathrm{N}(3)$ & $17(3)$ & $16(3)$ & $25(3)$ & $-5(2)$ & $4(2)$ & $-5(2)$ \\
$\mathrm{N}(4)$ & $10(2)$ & $18(3)$ & $24(3)$ & $0(2)$ & $0(2)$ & $-3(2)$ \\
$\mathrm{N}(5)$ & $13(3)$ & $18(3)$ & $27(3)$ & $-2(2)$ & $1(2)$ & $5(2)$ \\
$\mathrm{N}(6)$ & $8(2)$ & $18(3)$ & $31(3)$ & $2(2)$ & $1(2)$ & $0(2)$ \\
$\mathrm{N}(7)$ & $10(2)$ & $19(3)$ & $23(3)$ & $-4(2)$ & $1(2)$ & $-4(2)$ \\
$\mathrm{N}(8)$ & $9(2)$ & $20(3)$ & $25(3)$ & $-5(2)$ & $1(2)$ & $0(2)$ \\
$\mathrm{N}(9)$ & $16(3)$ & $20(3)$ & $24(3)$ & $0(2)$ & $-2(2)$ & $2(2)$ \\
$\mathrm{N}(10)$ & $22(3)$ & $17(3)$ & $29(3)$ & $-2(2)$ & $-5(2)$ & $0(2)$ \\
$\mathrm{N}(11)$ & $12(2)$ & $22(3)$ & $23(3)$ & $-2(2)$ & $0(2)$ & $-2(2)$ \\
$\mathrm{N}(12)$ & $13(2)$ & $13(3)$ & $26(3)$ & $0(2)$ & $1(2)$ & $-2(2)$ \\
$\mathrm{Cl}(1)$ & $33(1)$ & $43(1)$ & $47(1)$ & $-6(1)$ & $9(1)$ & $0(1)$ \\
$\mathrm{Cl}(2)$ & $61(1)$ & $39(1)$ & $47(1)$ & $-1(1)$ & $-3(1)$ & $-8(1)$ \\
$\mathrm{C}(1)$ & $11(3)$ & $21(3)$ & $26(3)$ & $2(3)$ & $2(2)$ & $5(3)$ \\
$\mathrm{C}(2)$ & $16(3)$ & $20(3)$ & $23(3)$ & $1(3)$ & $-3(3)$ & $2(3)$ \\
$\mathrm{C}(3)$ & $16(3)$ & $29(4)$ & $29(3)$ & $-2(3)$ & $0(3)$ & $-3(3)$ \\
$\mathrm{C}(4)$ & $33(4)$ & $27(4)$ & $39(4)$ & $-5(3)$ & $11(3)$ & $-9(3)$ \\
$\mathrm{C}(5)$ & $29(4)$ & $40(4)$ & $24(3)$ & $-4(3)$ & $8(3)$ & $-3(3)$ \\
$\mathrm{C}(6)$ & $13(3)$ & $15(3)$ & $31(3)$ & $-3(3)$ & $1(3)$ & $2(2)$ \\
$\mathrm{C}(7)$ & $41(4)$ & $22(4)$ & $20(3)$ & $-9(3)$ & $3(3)$ & $-2(3)$ \\
$\mathrm{C}(8)$ & $29(4)$ & $33(4)$ & $36(4)$ & $-12(3)$ & $0(3)$ & $-5(3)$ \\
$\mathrm{C}(9)$ & $21(3)$ & $17(3)$ & $33(4)$ & $-1(3)$ & $-4(3)$ & $-4(3)$ \\
$\mathrm{C}(10)$ & $8(3)$ & $19(3)$ & $24(3)$ & $0(3)$ & $5(2)$ & $-9(2)$ \\
$\mathrm{C}(11)$ & $8(3)$ & $21(3)$ & $33(4)$ & $1(3)$ & $2(3)$ & $-4(2)$ \\
$\mathrm{C}(12)$ & $17(3)$ & $24(4)$ & $33(4)$ & $-9(3)$ & $10(3)$ & $-7(3)$ \\
$\mathrm{C}(13)$ & $12(3)$ & $28(4)$ & $25(3)$ & $-5(3)$ & $1(3)$ & $-4(3)$ \\
$\mathrm{C}(14)$ & $16(3)$ & $24(4)$ & $28(3)$ & $6(3)$ & $0(3)$ & $-5(3)$ \\
$\mathrm{C}(15)$ & $6(3)$ & $20(3)$ & $26(3)$ & $0(3)$ & $3(2)$ & $-9(2)$ \\
& & & & & &
\end{tabular}




\begin{tabular}{|c|c|c|c|c|c|c|}
\hline$C(16)$ & $10(3)$ & $17(3)$ & $30(3)$ & $10(3)$ & $-2(3)$ & $2(2)$ \\
\hline$C(17)$ & $8(3)$ & $14(3)$ & $44(4)$ & $6(3)$ & $-1(3)$ & $1(2)$ \\
\hline$C(18)$ & $19(3)$ & $9(3)$ & $36(4)$ & $-2(3)$ & $12(3)$ & $-3(3)$ \\
\hline$C(19)$ & $11(3)$ & $20(3)$ & $28(3)$ & $-7(3)$ & $1(3)$ & $-5(3)$ \\
\hline$C(20)$ & $21(3)$ & $17(3)$ & $26(3)$ & $5(3)$ & $0(3)$ & $0(3)$ \\
\hline$C(21)$ & $24(4)$ & $18(3)$ & $28(3)$ & $4(3)$ & $2(3)$ & $5(3)$ \\
\hline $\mathrm{C}(22)$ & $16(3)$ & $24(4)$ & $31(4)$ & $2(3)$ & $-4(3)$ & $8(3)$ \\
\hline$C(23)$ & $12(3)$ & $28(4)$ & $34(4)$ & $-7(3)$ & $1(3)$ & $-5(3)$ \\
\hline$C(24)$ & $19(3)$ & $9(3)$ & $23(3)$ & $-3(2)$ & $1(3)$ & $4(2)$ \\
\hline$C(25)$ & $11(3)$ & $17(3)$ & $43(4)$ & $0(3)$ & $4(3)$ & $-1(3)$ \\
\hline$C(26)$ & $22(3)$ & $16(3)$ & $39(4)$ & $1(3)$ & $6(3)$ & $-8(3)$ \\
\hline$C(27)$ & $20(3)$ & $14(3)$ & $37(4)$ & $4(3)$ & $3(3)$ & $-3(3)$ \\
\hline $\mathrm{C}(28)$ & $17(3)$ & $18(3)$ & $19(3)$ & $-4(3)$ & $3(2)$ & $-4(3)$ \\
\hline$C(29)$ & $22(3)$ & $26(4)$ & $24(3)$ & $-2(3)$ & $7(3)$ & $2(3)$ \\
\hline$C(30)$ & $34(4)$ & $22(4)$ & $26(3)$ & $-5(3)$ & $10(3)$ & $-5(3)$ \\
\hline$C(31)$ & $21(4)$ & $32(4)$ & $34(4)$ & $-2(3)$ & $6(3)$ & $-11(3)$ \\
\hline$C(32)$ & $14(3)$ & $33(4)$ & $27(3)$ & $0(3)$ & $5(3)$ & $-2(3)$ \\
\hline$C(33)$ & $15(3)$ & $30(4)$ & $20(3)$ & $-9(3)$ & $4(2)$ & $-1(3)$ \\
\hline$C(34)$ & $11(3)$ & $22(4)$ & $38(4)$ & $-9(3)$ & $-2(3)$ & $1(3)$ \\
\hline$C(35)$ & $20(3)$ & $15(3)$ & $26(3)$ & $2(3)$ & $-1(3)$ & $9(3)$ \\
\hline$C(36)$ & $20(3)$ & $14(3)$ & $27(3)$ & $-3(3)$ & $-1(3)$ & $0(3)$ \\
\hline$C(37)$ & $11(3)$ & $22(3)$ & $24(3)$ & $6(3)$ & $5(2)$ & $-6(3)$ \\
\hline$C(38)$ & $21(3)$ & $14(3)$ & $24(3)$ & $1(3)$ & $3(3)$ & $-1(3)$ \\
\hline$C(39)$ & $21(3)$ & $24(4)$ & $28(3)$ & $5(3)$ & $2(3)$ & $5(3)$ \\
\hline$C(40)$ & $23(4)$ & $28(4)$ & $37(4)$ & $5(3)$ & $-6(3)$ & $5(3)$ \\
\hline$C(41)$ & $24(4)$ & $24(4)$ & $33(4)$ & $1(3)$ & $-2(3)$ & $-1(3)$ \\
\hline$C(42)$ & $14(3)$ & $24(4)$ & $23(3)$ & $3(3)$ & $-3(3)$ & $1(3)$ \\
\hline$C(43)$ & $26(3)$ & $20(3)$ & $16(3)$ & $-4(3)$ & $0(3)$ & $-3(3)$ \\
\hline$C(44)$ & $36(4)$ & $20(4)$ & $29(3)$ & $-9(3)$ & $4(3)$ & $-2(3)$ \\
\hline$C(45)$ & $21(3)$ & $16(3)$ & $33(3)$ & $-6(3)$ & $5(3)$ & $-2(3)$ \\
\hline$C(46)$ & $10(3)$ & $16(3)$ & $23(3)$ & $7(3)$ & $3(2)$ & $10(2)$ \\
\hline$C(47)$ & $14(3)$ & $19(3)$ & $23(3)$ & $1(3)$ & $5(3)$ & $-1(3)$ \\
\hline$C(48)$ & $14(3)$ & $21(3)$ & $29(3)$ & $-5(3)$ & $-1(3)$ & $2(3)$ \\
\hline C(49) & $17(3)$ & $28(4)$ & $19(3)$ & $3(3)$ & $-1(3)$ & $3(3)$ \\
\hline$C(50)$ & $10(3)$ & $21(3)$ & $28(3)$ & $4(3)$ & $4(3)$ & $1(3)$ \\
\hline $\mathrm{C}(51)$ & $12(3)$ & $14(3)$ & $29(3)$ & $2(3)$ & $1(3)$ & $-1(2)$ \\
\hline
\end{tabular}




\begin{tabular}{lllllll}
$\mathrm{C}(52)$ & $15(3)$ & $21(4)$ & $33(3)$ & $12(3)$ & $6(3)$ & $4(3)$ \\
$\mathrm{C}(53)$ & $15(3)$ & $19(3)$ & $38(4)$ & $7(3)$ & $3(3)$ & $-2(3)$ \\
$\mathrm{C}(54)$ & $16(3)$ & $11(3)$ & $36(4)$ & $0(3)$ & $-1(3)$ & $0(2)$ \\
$\mathrm{C}(55)$ & $52(5)$ & $50(5)$ & $46(5)$ & $-5(4)$ & $11(4)$ & $-8(4)$ \\
\hline
\end{tabular}

Table S 4. Hydrogen coordinates ( x 104) and isotropic displacement parameters ( $\AA 2$ x $10^{3}$ ).

\begin{tabular}{|c|c|c|c|c|}
\hline & $\mathrm{x}$ & $\mathrm{y}$ & z & $\mathrm{U}(\mathrm{eq})$ \\
\hline $\mathrm{H}(2)$ & 6474 & 2871 & 747 & 25 \\
\hline $\mathrm{H}(3)$ & 5560 & 2140 & 1340 & 30 \\
\hline $\mathrm{H}(4)$ & 5482 & 2640 & 2282 & 39 \\
\hline $\mathrm{H}(5)$ & 6292 & 3935 & 2625 & 37 \\
\hline $\mathrm{H}(7)$ & 7103 & 5355 & 2859 & 34 \\
\hline $\mathrm{H}(8)$ & 8153 & 6616 & 2703 & 40 \\
\hline $\mathrm{H}(9)$ & 8658 & 6397 & 1710 & 30 \\
\hline $\mathrm{H}(11)$ & 8360 & 2881 & 299 & 25 \\
\hline $\mathrm{H}(12)$ & 7957 & 2357 & -659 & 29 \\
\hline $\mathrm{H}(13)$ & 6854 & 3114 & -1387 & 27 \\
\hline $\mathrm{H}(14)$ & 6113 & 4389 & -1125 & 28 \\
\hline $\mathrm{H}(16)$ & 5323 & 5658 & -746 & 24 \\
\hline $\mathrm{H}(17)$ & 5033 & 6715 & 25 & 27 \\
\hline $\mathrm{H}(18)$ & 6189 & 6306 & 969 & 25 \\
\hline $\mathrm{H}(20)$ & 8493 & 2854 & 1597 & 26 \\
\hline $\mathrm{H}(21)$ & 10062 & 2282 & 1943 & 29 \\
\hline $\mathrm{H}(22)$ & 11504 & 2987 & 1784 & 30 \\
\hline $\mathrm{H}(23)$ & 11352 & 4309 & 1264 & 30 \\
\hline$H(25)$ & 11034 & 5605 & 639 & 28 \\
\hline $\mathrm{H}(26)$ & 9963 & 6720 & 41 & 31 \\
\hline $\mathrm{H}(27)$ & 8195 & 6382 & 109 & 29 \\
\hline $\mathrm{H}(29)$ & 7587 & 7914 & 1653 & 28 \\
\hline
\end{tabular}




\begin{tabular}{|c|c|c|c|c|}
\hline $\mathrm{H}(30)$ & 6231 & 7241 & 1935 & 32 \\
\hline $\mathrm{H}(31)$ & 4634 & 7833 & 1698 & 35 \\
\hline $\mathrm{H}(32)$ & 4388 & 9122 & 1170 & 30 \\
\hline $\mathrm{H}(34)$ & 4326 & 10535 & 636 & 29 \\
\hline $\mathrm{H}(35)$ & 4995 & 11706 & 80 & 25 \\
\hline $\mathrm{H}(36)$ & 6830 & 11401 & 134 & 25 \\
\hline $\mathrm{H}(38)$ & 9010 & 7888 & 786 & 24 \\
\hline $\mathrm{H}(39)$ & 10531 & 7370 & 1291 & 29 \\
\hline $\mathrm{H}(40)$ & 11411 & 8121 & 2105 & 37 \\
\hline $\mathrm{H}(41)$ & 10708 & 9375 & 2442 & 34 \\
\hline $\mathrm{H}(43)$ & 9695 & 10487 & 2815 & 25 \\
\hline $\mathrm{H}(44)$ & 8391 & 11619 & 2721 & 34 \\
\hline $\mathrm{H}(45)$ & 7237 & 11340 & 1747 & 28 \\
\hline $\mathrm{H}(47)$ & 6845 & 7881 & 312 & 22 \\
\hline $\mathrm{H}(48)$ & 6644 & 7361 & -657 & 26 \\
\hline H(49) & 7321 & 8101 & -1379 & 26 \\
\hline $\mathrm{H}(50)$ & 8224 & 9374 & -1117 & 23 \\
\hline $\mathrm{H}(52)$ & 9229 & 10645 & -753 & 27 \\
\hline $\mathrm{H}(53)$ & 9984 & 11724 & 13 & 29 \\
\hline $\mathrm{H}(54)$ & 9382 & 11346 & 963 & 26 \\
\hline $\mathrm{H}(55 \mathrm{~A})$ & 3504 & 4333 & 2692 & 59 \\
\hline $\mathrm{H}(55 \mathrm{~B})$ & 2817 & 4961 & 2239 & 59 \\
\hline
\end{tabular}

Table S 5. Atomic coordinates ( x 104) and equivalent isotropic displacement parameters ( $\mathrm{A} 2 \mathrm{x}$ 103)for Gapma. U(eq) is defined as one third of the trace of the orthogonalized Uij tensor.

\begin{tabular}{lccrc}
\hline & $\mathrm{x}$ & $\mathrm{y}$ & $\mathrm{z}$ & $\mathrm{U}(\mathrm{eq})$ \\
\hline $\mathrm{Ga}(1)$ & $4194(1)$ & $5806(1)$ & $806(1)$ & $33(1)$ \\
$\mathrm{N}(1)$ & $5185(1)$ & $5837(1)$ & $891(1)$ & $37(1)$ \\
$\mathrm{N}(2)$ & $4303(1)$ & $5841(1)$ & $1845(1)$ & $36(1)$ \\
$\mathrm{N}(3)$ & $3233(1)$ & $6767(1)$ & $1767(1)$ & $41(1)$
\end{tabular}




\begin{tabular}{lllrl}
$\mathrm{C}(1)$ & $5723(1)$ & $5870(1)$ & $500(1)$ & $45(1)$ \\
$\mathrm{C}(2)$ & $6295(1)$ & $5883(1)$ & $884(1)$ & $53(1)$ \\
$\mathrm{C}(3)$ & $6096(1)$ & $5862(1)$ & $1540(1)$ & $50(1)$ \\
$\mathrm{C}(4)$ & $5412(1)$ & $5833(1)$ & $1531(1)$ & $40(1)$ \\
$\mathrm{C}(5)$ & $4912(1)$ & $5825(1)$ & $2027(1)$ & $40(1)$ \\
$\mathrm{C}(6)$ & $3776(1)$ & $5842(1)$ & $2341(1)$ & $42(1)$ \\
$\mathrm{C}(7)$ & $3468(1)$ & $6523(1)$ & $2394(1)$ & $44(1)$ \\
\hline
\end{tabular}

Table S 6. Bond lengths $[\AA]$ and angles $\left[^{\circ}\right]$ for Gapma.

\begin{tabular}{ll}
\hline $\mathrm{Ga}(1)-\mathrm{N}(1)$ & $2.0144(13)$ \\
$\mathrm{Ga}(1)-\mathrm{N}(1) \# 1$ & $2.0144(13)$ \\
$\mathrm{Ga}(1)-\mathrm{N}(1) \# 2$ & $2.0144(13)$ \\
$\mathrm{Ga}(1)-\mathrm{N}(2) \# 2$ & $2.1148(14)$ \\
$\mathrm{Ga}(1)-\mathrm{N}(2)$ & $2.1148(14)$ \\
$\mathrm{Ga}(1)-\mathrm{N}(2) \# 1$ & $2.1148(14)$ \\
$\mathrm{N}(1)-\mathrm{C}(1)$ & $1.347(2)$ \\
$\mathrm{N}(1)-\mathrm{C}(4)$ & $1.374(2)$ \\
$\mathrm{N}(2)-\mathrm{C}(5)$ & $1.288(2)$ \\
$\mathrm{N}(2)-\mathrm{C}(6)$ & $1.464(2)$ \\
$\mathrm{N}(3)-\mathrm{C}(7) \# 2$ & $1.442(2)$ \\
$\mathrm{N}(3)-\mathrm{C}(7)$ & $1.442(2)$ \\
$\mathrm{N}(3)-\mathrm{C}(7) \# 1$ & $1.442(2)$ \\
$\mathrm{C}(1)-\mathrm{C}(2)$ & $1.395(3)$ \\
$\mathrm{C}(2)-\mathrm{C}(3)$ & $1.390(3)$ \\
$\mathrm{C}(3)-\mathrm{C}(4)$ & $1.385(3)$ \\
$\mathrm{C}(4)-\mathrm{C}(5)$ & $1.425(2)$ \\
$\mathrm{C}(6)-\mathrm{C}(7)$ & $1.516(3)$ \\
$\mathrm{N}(1)-\mathrm{Ga}(1)-\mathrm{N}(1) \# 1$ & $96.53(5)$ \\
$\mathrm{N}(1)-\mathrm{Ga}(1)-\mathrm{N}(1) \# 2$ & $96.53(5)$ \\
$\mathrm{N}(1) \# 1-\mathrm{Ga}(1)-\mathrm{N}(1) \# 2$ & $96.53(5)$ \\
$\mathrm{N}(1)-\mathrm{Ga}(1)-\mathrm{N}(2) \# 2$ & $90.63(5)$ \\
& \\
& \\
& \\
&
\end{tabular}




\begin{tabular}{lr}
$\mathrm{N}(1) \# 1-\mathrm{Ga}(1)-\mathrm{N}(2) \# 2$ & $172.01(5)$ \\
$\mathrm{N}(1) \# 2-\mathrm{Ga}(1)-\mathrm{N}(2) \# 2$ & $79.09(5)$ \\
$\mathrm{N}(1)-\mathrm{Ga}(1)-\mathrm{N}(2)$ & $79.09(5)$ \\
$\mathrm{N}(1) \# 1-\mathrm{Ga}(1)-\mathrm{N}(2)$ & $90.63(5)$ \\
$\mathrm{N}(1) \# 2-\mathrm{Ga}(1)-\mathrm{N}(2)$ & $172.01(5)$ \\
$\mathrm{N}(2) \# 2-\mathrm{Ga}(1)-\mathrm{N}(2)$ & $94.20(5)$ \\
$\mathrm{N}(1)-\mathrm{Ga}(1)-\mathrm{N}(2) \# 1$ & $172.01(5)$ \\
$\mathrm{N}(1) \# 1-\mathrm{Ga}(1)-\mathrm{N}(2) \# 1$ & $79.09(5)$ \\
$\mathrm{N}(1) \# 2-\mathrm{Ga}(1)-\mathrm{N}(2) \# 1$ & $90.63(5)$ \\
$\mathrm{N}(2) \# 2-\mathrm{Ga}(1)-\mathrm{N}(2) \# 1$ & $94.20(5)$ \\
$\mathrm{N}(2)-\mathrm{Ga}(1)-\mathrm{N}(2) \# 1$ & $94.20(5)$ \\
$\mathrm{C}(1)-\mathrm{N}(1)-\mathrm{C}(4)$ & $106.55(15)$ \\
$\mathrm{C}(1)-\mathrm{N}(1)-\mathrm{Ga}(1)$ & $139.06(13)$ \\
$\mathrm{C}(4)-\mathrm{N}(1)-\mathrm{Ga}(1)$ & $114.39(12)$ \\
$\mathrm{C}(5)-\mathrm{N}(2)-\mathrm{C}(6)$ & $120.08(15)$ \\
$\mathrm{C}(5)-\mathrm{N}(2)-\mathrm{Ga}(1)$ & $112.52(12)$ \\
$\mathrm{C}(6)-\mathrm{N}(2)-\mathrm{Ga}(1)$ & $127.30(12)$ \\
$\mathrm{C}(7) \# 2-\mathrm{N}(3)-\mathrm{C}(7)$ & $118.59(5)$ \\
$\mathrm{C}(7) \# 2-\mathrm{N}(3)-\mathrm{C}(7) \# 1$ & $118.59(5)$ \\
$\mathrm{C}(7)-\mathrm{N}(3)-\mathrm{C}(7) \# 1$ & $118.59(5)$ \\
$\mathrm{N}(1)-\mathrm{C}(1)-\mathrm{C}(2)$ & $110.11(18)$ \\
$\mathrm{C}(3)-\mathrm{C}(2)-\mathrm{C}(1)$ & $106.91(17)$ \\
$\mathrm{C}(4)-\mathrm{C}(3)-\mathrm{C}(2)$ & $106.16(18)$ \\
$\mathrm{N}(1)-\mathrm{C}(4)-\mathrm{C}(3)$ & $110.27(17)$ \\
$\mathrm{N}(1)-\mathrm{C}(4)-\mathrm{C}(5)$ & $115.25(16)$ \\
$\mathrm{C}(3)-\mathrm{C}(4)-\mathrm{C}(5)$ & $134.42(18)$ \\
$\mathrm{N}(2)-\mathrm{C}(5)-\mathrm{C}(4)$ & $118.58(16)$ \\
$\mathrm{N}(2)-\mathrm{C}(6)-\mathrm{C}(7)$ & $110.44(16)$ \\
$\mathrm{N}(3)-\mathrm{C}(7)-\mathrm{C}(6)$ & $112.63(18)$ \\
& \\
\hline
\end{tabular}

Table S 7. Anisotropic displacement parameters ( $\AA 2$ x 103) for Gapma . The anisotropic displacement factor exponent takes the form: $-2 \quad 2\left[\mathrm{~h} 2 \mathrm{a} * 2 \mathrm{U} 11+\ldots+2 \mathrm{hk} \mathrm{a} \mathrm{b}^{*} \mathrm{U} 12\right]$. 


\begin{tabular}{lllllll}
\hline & $\mathrm{U}^{11}$ & $\mathrm{U}^{22}$ & $\mathrm{U}^{33}$ & $\mathrm{U}^{23}$ & $\mathrm{U}^{13}$ & $\mathrm{U}^{12}$ \\
\hline $\mathrm{Ga}(1)$ & $33(1)$ & $33(1)$ & $33(1)$ & $-2(1)$ & $2(1)$ & $2(1)$ \\
$\mathrm{N}(1)$ & $35(1)$ & $36(1)$ & $40(1)$ & $-7(1)$ & $1(1)$ & $3(1)$ \\
$\mathrm{N}(2)$ & $41(1)$ & $36(1)$ & $33(1)$ & $-1(1)$ & $2(1)$ & $4(1)$ \\
$\mathrm{N}(3)$ & $41(1)$ & $41(1)$ & $41(1)$ & $-3(1)$ & $3(1)$ & $3(1)$ \\
$\mathrm{C}(1)$ & $44(1)$ & $42(1)$ & $49(1)$ & $-10(1)$ & $10(1)$ & $1(1)$ \\
$\mathrm{C}(2)$ & $37(1)$ & $50(1)$ & $72(2)$ & $-10(1)$ & $6(1)$ & $5(1)$ \\
$\mathrm{C}(3)$ & $41(1)$ & $46(1)$ & $62(1)$ & $-2(1)$ & $-8(1)$ & $5(1)$ \\
$\mathrm{C}(4)$ & $40(1)$ & $35(1)$ & $45(1)$ & $-3(1)$ & $-5(1)$ & $6(1)$ \\
$\mathrm{C}(5)$ & $49(1)$ & $36(1)$ & $35(1)$ & $-1(1)$ & $-6(1)$ & $5(1)$ \\
$\mathrm{C}(6)$ & $45(1)$ & $49(1)$ & $33(1)$ & $1(1)$ & $5(1)$ & $2(1)$ \\
$\mathrm{C}(7)$ & $48(1)$ & $47(1)$ & $36(1)$ & $-8(1)$ & $9(1)$ & $2(1)$ \\
& & & & & & \\
\hline
\end{tabular}

Table S 8. Hydrogen coordinates ( x 104) and isotropic displacement parameters $(\AA 2$ x 103 ) for Gapma.

\begin{tabular}{lrrrr}
\hline & $x$ & $y$ & $z$ & $U(e q)$ \\
& & & & \\
$H(1)$ & 5714 & 5883 & 40 & 54 \\
H(2) & 6728 & 5902 & 729 & 64 \\
H(3) & 6367 & 5867 & 1912 & 60 \\
H(5) & 5024 & 5809 & 2472 & 48 \\
H(6A) & 3956 & 5714 & 2766 & 51 \\
H(6B) & 3440 & 5523 & 2219 & 51 \\
H(7A) & 3101 & 6505 & 2702 & 52 \\
H(7B) & 3792 & 6828 & 2569 & 52 \\
\hline
\end{tabular}

Table S 9. Atomic coordinates ( x 104) and equivalent isotropic displacement parameters ( $\AA 2 \mathrm{x}$ 103) for $\mathrm{Co}(\mathrm{ppz} 2) \mathrm{Cl} 2 . \mathrm{U}(e q)$ is defined as one third of the trace of the orthogonalized Uij tensor. 


\begin{tabular}{|c|c|c|c|c|}
\hline & $\mathrm{X}$ & $\mathrm{y}$ & z & $\mathrm{U}(\mathrm{eq})$ \\
\hline $\operatorname{Co}(1)$ & $1345(1)$ & $7925(1)$ & $9072(1)$ & $43(1)$ \\
\hline $\mathrm{N}(1)$ & $3736(3)$ & $8375(3)$ & $9676(2)$ & $44(1)$ \\
\hline $\mathrm{N}(2)$ & $5135(3)$ & $8688(2)$ & $9188(2)$ & $42(1)$ \\
\hline $\mathrm{N}(3)$ & $963(3)$ & $6549(3)$ & $7880(2)$ & $52(1)$ \\
\hline $\mathrm{N}(4)$ & $1717(4)$ & $5203(3)$ & $7755(2)$ & $59(1)$ \\
\hline $\mathrm{Cl}(1)$ & $176(1)$ & $10117(1)$ & $8502(1)$ & $57(1)$ \\
\hline $\mathrm{Cl}(2)$ & $189(1)$ & $6732(1)$ & $10314(1)$ & $63(1)$ \\
\hline$C(1)$ & $4837(4)$ & $6998(3)$ & $7753(2)$ & $47(1)$ \\
\hline$C(2)$ & $4779(4)$ & $6820(4)$ & $6722(2)$ & $61(1)$ \\
\hline$C(3)$ & $4983(5)$ & $8067(5)$ & $6112(2)$ & $68(1)$ \\
\hline$C(4)$ & $5258(5)$ & $9456(5)$ & $6514(3)$ & $70(1)$ \\
\hline$C(5)$ & $5310(4)$ & $9669(4)$ & $7534(2)$ & $58(1)$ \\
\hline$C(6)$ & $5099(3)$ & $8444(3)$ & $8133(2)$ & $44(1)$ \\
\hline$C(7)$ & $3216(4)$ & $4740(3)$ & $8354(2)$ & $56(1)$ \\
\hline$C(8)$ & $3155(6)$ & $3405(4)$ & $8880(3)$ & $81(1)$ \\
\hline$C(9)$ & $4588(9)$ & $2922(4)$ & $9446(4)$ & $101(2)$ \\
\hline$C(10)$ & $6036(7)$ & $3790(5)$ & $9517(3)$ & $92(1)$ \\
\hline$C(11)$ & $6119(5)$ & $5138(4)$ & $8995(3)$ & $69(1)$ \\
\hline$C(12)$ & $4689(4)$ & $5625(3)$ & $8387(2)$ & $52(1)$ \\
\hline$C(13)$ & $4206(4)$ & $8724(3)$ & $10627(2)$ & $50(1)$ \\
\hline$C(14)$ & $5864(4)$ & $9254(3)$ & $10736(2)$ & $53(1)$ \\
\hline$C(15)$ & $6422(4)$ & $9208(3)$ & $9811(2)$ & $49(1)$ \\
\hline$C(16)$ & $-438(5)$ & $6570(4)$ & $7242(2)$ & $66(1)$ \\
\hline$C(17)$ & $-599(6)$ & $5238(6)$ & $6724(4)$ & $91(1)$ \\
\hline$C(18)$ & $789(5)$ & $4398(5)$ & $7062(4)$ & $89(1)$ \\
\hline
\end{tabular}

Table S 10. Bond lengths $[\AA]$ and angles $\left[^{\circ}\right]$ for $\mathrm{Co}(\mathrm{ppz} 2) \mathrm{Cl} 2$.

\begin{tabular}{ll}
\hline $\mathrm{Co}(1)-\mathrm{N}(1)$ & $2.025(3)$ \\
$\mathrm{Co}(1)-\mathrm{N}(3)$ & $2.031(3)$
\end{tabular}




$\begin{array}{ll}\mathrm{Co}(1)-\mathrm{Cl}(2) & 2.2531(13) \\ \mathrm{Co}(1)-\mathrm{Cl}(1) & 2.2558(14) \\ \mathrm{N}(1)-\mathrm{C}(13) & 1.351(4) \\ \mathrm{N}(1)-\mathrm{N}(2) & 1.363(3) \\ \mathrm{N}(2)-\mathrm{C}(15) & 1.343(3) \\ \mathrm{N}(2)-\mathrm{C}(6) & 1.451(4) \\ \mathrm{N}(3)-\mathrm{C}(16) & 1.342(4) \\ \mathrm{N}(3)-\mathrm{N}(4) & 1.348(4) \\ \mathrm{N}(4)-\mathrm{C}(18) & 1.345(4) \\ \mathrm{N}(4)-\mathrm{C}(7) & 1.434(4) \\ \mathrm{C}(1)-\mathrm{C}(6) & 1.390(4) \\ \mathrm{C}(1)-\mathrm{C}(2) & 1.410(4) \\ \mathrm{C}(1)-\mathrm{C}(12) & 1.502(4) \\ \mathrm{C}(2)-\mathrm{C}(3) & 1.399(6) \\ \mathrm{C}(3)-\mathrm{C}(4) & 1.356(6) \\ \mathrm{C}(4)-\mathrm{C}(5) & 1.399(5) \\ \mathrm{C}(5)-\mathrm{C}(6) & 1.375(4) \\ \mathrm{C}(7)-\mathrm{C}(8) & 1.385(5) \\ \mathrm{C}(7)-\mathrm{C}(12) & 1.396(4) \\ \mathrm{C}(8)-\mathrm{C}(9) & 1.376(7) \\ \mathrm{C}(9)-\mathrm{C}(10) & 1.370(8) \\ \mathrm{C}(10)-\mathrm{C}(11) & 1.394(6) \\ \mathrm{C}(11)-\mathrm{C}(12) & 1.404(4) \\ \mathrm{C}(13)-\mathrm{C}(14) & 1.382(5) \\ \mathrm{C}(14)-\mathrm{C}(15) & 1.370(4) \\ \mathrm{C}(16)-\mathrm{C}(17) & 1.375(6) \\ \mathrm{C}(17)-\mathrm{C}(18) & 1.367(6) \\ \mathrm{N}(1)-\mathrm{Co}(1)-\mathrm{N}(3) & 120.59(10) \\ \mathrm{N}(1)-\mathrm{Co}(1)-\mathrm{Cl}(2) & 102.09(8) \\ \mathrm{N}(3)-\mathrm{Co}(1)-\mathrm{Cl}(2) & 106.13(9) \\ \mathrm{N}(1)-\mathrm{Co}(1)-\mathrm{Cl}(1) & 107.83(8) \\ \mathrm{N}(3)-\mathrm{Co}(1)-\mathrm{Cl}(1) & 102.40(9) \\ \mathrm{Cl}(2)-\mathrm{Co}(1)-\mathrm{Cl}(1) & 118.84(4) \\ \mathrm{C}(13)-\mathrm{N}(1)-\mathrm{N}(2) & 104.9(2) \\ \mathrm{C}(13)-\mathrm{N}(1)-\mathrm{Co}(1) & 126.9(2) \\ & \end{array}$




$\begin{array}{ll}\mathrm{N}(2)-\mathrm{N}(1)-\mathrm{Co}(1) & 127.07(16) \\ \mathrm{C}(15)-\mathrm{N}(2)-\mathrm{N}(1) & 111.0(2) \\ \mathrm{C}(15)-\mathrm{N}(2)-\mathrm{C}(6) & 128.6(2) \\ \mathrm{N}(1)-\mathrm{N}(2)-\mathrm{C}(6) & 120.5(2) \\ \mathrm{C}(16)-\mathrm{N}(3)-\mathrm{N}(4) & 106.0(3) \\ \mathrm{C}(16)-\mathrm{N}(3)-\mathrm{Co}(1) & 124.3(2) \\ \mathrm{N}(4)-\mathrm{N}(3)-\mathrm{Co}(1) & 126.6(2) \\ \mathrm{C}(18)-\mathrm{N}(4)-\mathrm{N}(3) & 109.9(3) \\ \mathrm{C}(18)-\mathrm{N}(4)-\mathrm{C}(7) & 128.1(3) \\ \mathrm{N}(3)-\mathrm{N}(4)-\mathrm{C}(7) & 121.9(2) \\ \mathrm{C}(6)-\mathrm{C}(1)-\mathrm{C}(2) & 117.7(3) \\ \mathrm{C}(6)-\mathrm{C}(1)-\mathrm{C}(12) & 123.2(3) \\ \mathrm{C}(2)-\mathrm{C}(1)-\mathrm{C}(12) & 119.0(3) \\ \mathrm{C}(3)-\mathrm{C}(2)-\mathrm{C}(1) & 120.5(3) \\ \mathrm{C}(4)-\mathrm{C}(3)-\mathrm{C}(2) & 120.0(3) \\ \mathrm{C}(3)-\mathrm{C}(4)-\mathrm{C}(5) & 120.8(3) \\ \mathrm{C}(6)-\mathrm{C}(5)-\mathrm{C}(4) & 119.2(3) \\ \mathrm{C}(5)-\mathrm{C}(6)-\mathrm{C}(1) & 121.8(3) \\ \mathrm{C}(5)-\mathrm{C}(6)-\mathrm{N}(2) & 118.5(3) \\ \mathrm{C}(1)-\mathrm{C}(6)-\mathrm{N}(2) & 119.7(2) \\ \mathrm{C}(8)-\mathrm{C}(7)-\mathrm{C}(12) & 121.9(3) \\ \mathrm{C}(8)-\mathrm{C}(7)-\mathrm{N}(4) & 118.3(3) \\ \mathrm{C}(12)-\mathrm{C}(7)-\mathrm{N}(4) & 119.8(3) \\ \mathrm{C}(9)-\mathrm{C}(8)-\mathrm{C}(7) & 119.4(4) \\ \mathrm{C}(10)-\mathrm{C}(9)-\mathrm{C}(8) & 120.0(4) \\ \mathrm{C}(9)-\mathrm{C}(10)-\mathrm{C}(11) & 121.4(4) \\ \mathrm{C}(10)-\mathrm{C}(11)-\mathrm{C}(12) & 119.5(4) \\ \mathrm{C}(7)-\mathrm{C}(12)-\mathrm{C}(11) & 117.7(3) \\ \mathrm{C}(7)-\mathrm{C}(12)-\mathrm{C}(1) & 122.8(3) \\ \mathrm{C}(11)-\mathrm{C}(12)-\mathrm{C}(1) & 119.3(3) \\ \mathrm{N}(1)-\mathrm{C}(13)-\mathrm{C}(14) & 110.8(3) \\ \mathrm{C}(15)-\mathrm{C}(14)-\mathrm{C}(13) & 105.5(3) \\ \mathrm{N}(2)-\mathrm{C}(15)-\mathrm{C}(14) & 107.8(3) \\ \mathrm{N}(4)-\mathrm{C}(18)-\mathrm{C}(17) & 10.7(4) \\ & \end{array}$


Table S 11. Anisotropic displacement parameters $(\AA 2 \times 103)$ for $\mathrm{Co}(\mathrm{ppz} 2) \mathrm{Cl} 2$. The anisotropic displacement factor exponent takes the form: $-2 \quad 2\left[\mathrm{~h} 2 \mathrm{a}^{*} 2 \mathrm{U} 11+\ldots+2 \mathrm{hk} \mathrm{a} \mathrm{b}^{*} \mathrm{U} 12\right]$.

\begin{tabular}{|c|c|c|c|c|c|c|}
\hline & $\mathrm{U}^{11}$ & $\mathrm{U}^{22}$ & $\mathrm{U}^{33}$ & $\mathrm{U}^{23}$ & $\mathrm{U}^{13}$ & $\mathrm{U}^{12}$ \\
\hline $\operatorname{Co}(1)$ & $36(1)$ & $45(1)$ & $46(1)$ & $-1(1)$ & 1(1) & $-3(1)$ \\
\hline $\mathrm{N}(1)$ & $36(1)$ & $53(1)$ & $43(1)$ & $-2(1)$ & 2(1) & $-2(1)$ \\
\hline $\mathrm{N}(2)$ & $35(1)$ & $45(1)$ & $44(1)$ & $-2(1)$ & $3(1)$ & $-4(1)$ \\
\hline $\mathrm{N}(3)$ & $47(2)$ & $57(1)$ & $52(1)$ & $-8(1)$ & $-3(1)$ & $0(1)$ \\
\hline $\mathrm{N}(4)$ & $58(2)$ & $46(1)$ & 71(2) & $-14(1)$ & $-7(1)$ & $-7(1)$ \\
\hline $\mathrm{Cl}(1)$ & $60(1)$ & $48(1)$ & $64(1)$ & $9(1)$ & $6(1)$ & $2(1)$ \\
\hline $\mathrm{Cl}(2)$ & $58(1)$ & $63(1)$ & $68(1)$ & $17(1)$ & $8(1)$ & $-11(1)$ \\
\hline $\mathrm{C}(1)$ & $36(2)$ & $57(2)$ & $47(1)$ & $-5(1)$ & $1(1)$ & $7(1)$ \\
\hline$C(2)$ & $50(2)$ & $85(2)$ & $47(2)$ & $-13(2)$ & $-3(1)$ & $3(2)$ \\
\hline$C(3)$ & $52(2)$ & $109(3)$ & $43(2)$ & $2(2)$ & $2(1)$ & $-5(2)$ \\
\hline$C(4)$ & $53(2)$ & $100(3)$ & $57(2)$ & $23(2)$ & $0(2)$ & $-15(2)$ \\
\hline $\mathrm{C}(5)$ & $48(2)$ & $62(2)$ & $64(2)$ & $8(1)$ & $6(1)$ & $-8(1)$ \\
\hline$C(6)$ & $31(1)$ & $57(2)$ & $45(1)$ & $1(1)$ & $3(1)$ & $-1(1)$ \\
\hline$C(7)$ & $66(2)$ & 41(1) & $60(2)$ & $-9(1)$ & $-5(2)$ & $3(1)$ \\
\hline $\mathrm{C}(8)$ & $106(3)$ & $45(2)$ & $89(3)$ & $-4(2)$ & $-5(2)$ & $-6(2)$ \\
\hline C(9) & $153(5)$ & $50(2)$ & $96(3)$ & $15(2)$ & $-15(3)$ & $16(3)$ \\
\hline$C(10)$ & $105(4)$ & 78(3) & $85(2)$ & $8(2)$ & $-24(3)$ & $35(3)$ \\
\hline $\mathrm{C}(11)$ & $68(3)$ & 71(2) & $65(2)$ & $-6(2)$ & $-13(2)$ & $14(2)$ \\
\hline$C(12)$ & $56(2)$ & $44(1)$ & $54(2)$ & $-9(1)$ & $-2(1)$ & $9(1)$ \\
\hline$C(13)$ & $52(2)$ & $56(2)$ & $42(1)$ & $0(1)$ & $3(1)$ & $4(1)$ \\
\hline$C(14)$ & $46(2)$ & $58(2)$ & $51(1)$ & $-3(1)$ & $-9(1)$ & $3(1)$ \\
\hline$C(15)$ & $36(2)$ & $51(2)$ & $58(2)$ & $-2(1)$ & $-1(1)$ & $-7(1)$ \\
\hline$C(16)$ & $49(2)$ & $82(2)$ & $66(2)$ & $-9(2)$ & $-7(2)$ & $-5(2)$ \\
\hline$C(17)$ & 74(3) & $101(3)$ & $92(3)$ & $-31(2)$ & $-27(2)$ & $-11(2)$ \\
\hline$C(18)$ & $86(3)$ & $73(2)$ & $104(3)$ & $-40(2)$ & $-23(2)$ & $-9(2)$ \\
\hline
\end{tabular}


Table S 12. Hydrogen coordinates ( x 104) and isotropic displacement parameters ( $\AA 2$ x 103$)$.

\begin{tabular}{lrrrr}
\hline & $x$ & $y$ & $z$ & $U(e q)$ \\
& & & & \\
$H(2)$ & 4605 & 5866 & 6445 & 73 \\
$H(3)$ & 4930 & 7943 & 5432 & 82 \\
$\mathrm{H}(4)$ & 5413 & 10278 & 6106 & 84 \\
$\mathrm{H}(5)$ & 5486 & 10627 & 7804 & 70 \\
$\mathrm{H}(8)$ & 2155 & 2840 & 8851 & 97 \\
$\mathrm{H}(9)$ & 4573 & 2007 & 9781 & 122 \\
$\mathrm{H}(10)$ & 6983 & 3472 & 9921 & 110 \\
$\mathrm{H}(11)$ & 7114 & 5710 & 9049 & 83 \\
$\mathrm{H}(13)$ & 3507 & 8621 & 11140 & 60 \\
$\mathrm{H}(14)$ & 6474 & 9574 & 11315 & 63 \\
$\mathrm{H}(15)$ & 7499 & 9488 & 9644 & 58 \\
$\mathrm{H}(16)$ & -1195 & 7376 & 7162 & 79 \\
$\mathrm{H}(17)$ & -1465 & 4967 & 6248 & 109 \\
$\mathrm{H}(18)$ & 1047 & 3434 & 6849 & 107 \\
& & & & \\
\hline
\end{tabular}


